\title{
CALDERO-CHAPOTON ALGEBRAS
}

\author{
GIOVANNI CERULLI IRELLI, DANIEL LABARDINI-FRAGOSO, AND JAN SCHRÖER
}

\begin{abstract}
Motivated by the representation theory of quivers with potential introduced by Derksen, Weyman and Zelevinsky and by work of Caldero and Chapoton, who gave explicit formulae for the cluster variables of cluster algebras of Dynkin type, we associate a Caldero-Chapoton algebra $\mathcal{A}_{\Lambda}$ to any (possibly infinite-dimensional) basic algebra $\Lambda$. By definition, $\mathcal{A}_{\Lambda}$ is (as a vector space) generated by the Caldero-Chapoton functions $C_{\Lambda}(\mathcal{M})$ of the decorated representations $\mathcal{M}$ of $\Lambda$. If $\Lambda=\mathcal{P}(Q, W)$ is the Jacobian algebra defined by a 2 -acyclic quiver $Q$ with non-degenerate potential $W$, then we have $\mathcal{A}_{Q} \subseteq \mathcal{A}_{\Lambda} \subseteq \mathcal{A}_{Q}^{\text {up }}$, where $\mathcal{A}_{Q}$ and $\mathcal{A}_{Q}^{\text {up }}$ are the cluster algebra and the upper cluster algebra associated to $Q$. The set $\mathcal{B}_{\Lambda}$ of generic Caldero-Chapoton functions is parametrized by the strongly reduced components of the varieties of representations of the Jacobian algebra $\mathcal{P}(Q, W)$ and was introduced by Geiss, Leclerc and Schröer. Plamondon parametrized the strongly reduced components for finite-dimensional basic algebras. We generalize this to arbitrary basic algebras. Furthermore, we prove a decomposition theorem for strongly reduced components. We define $\mathcal{B}_{\Lambda}$ for arbitrary $\Lambda$, and we conjecture that $\mathcal{B}_{\Lambda}$ is a basis of the Caldero-Chapoton algebra $\mathcal{A}_{\Lambda}$. Thanks to the decomposition theorem, all elements of $\mathcal{B}_{\Lambda}$ can be seen as generalized cluster monomials. As another application, we obtain a new proof for the sign-coherence of $g$-vectors.
\end{abstract}

\section{Contents}

1. Introduction

2. Basic algebras and decorated representations

3. $E$-invariants and $g$-vectors of decorated representations

4. Caldero-Chapoton algebras

5. Strongly reduced components of representation varieties

6. Component graphs and $C C$-clusters

7. Caldero-Chapoton algebras and cluster algebras

8. Sign-coherence of generic $g$-vectors

9. Examples

Acknowledgements

References

\section{INTRODUCTION}

1.1. Let $\mathcal{A}_{Q}$ be the Fomin-Zelevinsky cluster algebra [FZ1, FZ2 associated to a finite 2-acyclic quiver $Q$. By definition $\mathcal{A}_{Q}$ is generated by an inductively defined set of rational functions, called cluster variables. The cluster variables are contained

Received by the editors December 4, 2012 and, in revised form, April 30, 2013.

2010 Mathematics Subject Classification. Primary 13F60; Secondary 16G10, 16G20. 
in the set $\mathcal{M}_{Q}$ of cluster monomials, which are by definition certain monomials in the cluster variables.

Now let $W$ be a non-degenerate potential for $Q$, and let $\Lambda=\mathcal{P}(Q, W)$ be the associated Jacobian algebra introduced by Derksen, Weyman and Zelevinsky DWZ1, DWZ2. The category of decorated representations of $\Lambda$ is denoted by $\operatorname{decrep}(\Lambda)$. To any $\mathcal{M} \in \operatorname{decrep}(\Lambda)$ one can associate a Laurent polynomial $C_{\Lambda}(\mathcal{M})$, the Caldero-Chapoton function of $\mathcal{M}$. It follows from DWZ1, DWZ2 that the cluster monomials form a subset of the set $\mathcal{C}_{\Lambda}$ of Caldero-Chapoton functions.

1.2. The generic basis conjecture. One of the main problems in cluster algebra theory is to find a basis of $\mathcal{A}_{Q}$ with favourable properties. As an important requirement, this basis should contain the set $\mathcal{M}_{Q}$ of cluster monomials in a natural way.

The concept of strongly reduced irreducible components of varieties of decorated representations of a Jacobian algebra $\Lambda$ was introduced in GLS. To each strongly reduced component $Z$ one can associate a generic Caldero-Chapoton function $C_{\Lambda}(Z)$; see Sections 4.1 and 6.4. It was conjectured in GLS that the set $\mathcal{B}_{\Lambda}$ of generic Caldero-Chapoton functions forms a $\mathbb{C}$-basis of $\mathcal{A}_{Q}$. Using a non-degenerate potential defined by Labardini [La1, La2, Plamondon [P2] found a counterexample and then conjectured that $\mathcal{B}_{\Lambda}$ is a basis of the upper cluster algebra $\mathcal{A}_{Q}^{\text {up }}$. This conjecture should also be wrong in general. We replace it by yet another conjecture.

We study the Caldero-Chapoton algebra

$$
\mathcal{A}_{\Lambda}:=\left\langle C_{\Lambda}(\mathcal{M}) \mid \mathcal{M} \in \operatorname{decrep}(\Lambda)\right\rangle_{\text {alg }}
$$

generated by all Caldero-Chapoton functions. We do not restrict ourselves to Jacobian algebras, but work with algebras $\Lambda$ defined as arbitrary quotients of completed path algebras. In particular, we generalize the notion of a Caldero-Chapoton function to this general setup. One easily checks that the functions $C_{\Lambda}(\mathcal{M})$ do not only generate $\mathcal{A}_{\Lambda}$ as an algebra but also as a vector space over the ground field $\mathbb{C}$.

Conjecture 1.1. $\mathcal{B}_{\Lambda}$ is a $\mathbb{C}$-basis of $\mathcal{A}_{\Lambda}$.

We show that the set $\mathcal{B}_{\Lambda}$ of generic Caldero-Chapoton functions is linearly independent provided the kernel of the skew-symmetric incidence matrix $B_{Q}$ of $Q$ does not contain any non-zero element in $\mathbb{Q}_{>0}^{n}$. This generalizes [P2, Proposition 3.19].

For $\Lambda=\mathcal{P}(Q, W)$, a Jacobian algebra associated to a quiver $Q$ with nondegenerate potential $W$, we have

$$
\mathcal{A}_{Q} \subseteq \mathcal{A}_{\Lambda} \subseteq \mathcal{A}_{Q}^{\mathrm{up}}
$$

where $\mathcal{A}_{Q}$ is the cluster algebra and $\mathcal{A}_{Q}^{\text {up }}$ is the upper cluster algebra associated to $Q$. (We refer to BFZ, DWZ1, FZ1 for missing definitions.) For this special case, we give a list of open problems, which hopefully will lead to a better understanding of the rather mysterious relation between $\mathcal{A}_{Q}$ and $\mathcal{A}_{Q}^{\text {up }}$.

1.3. Parametrization of strongly reduced components. Plamondon P2, Theorem 1.2] parametrized the strongly reduced components for finite-dimensional basic algebras. (For our (non-standard) definition of a basic algebra we refer to Section [2.1) We generalize Plamondon's result to arbitrary basic algebras. Let $\Lambda=\mathbb{C}\langle\langle Q\rangle\rangle / I$ be a basic algebra, where the quiver $Q$ has $n$ vertices. Let $\operatorname{dec} \operatorname{Irr}(\Lambda)$ be the set of irreducible components of all varieties $\operatorname{decrep}_{\mathbf{d}, \mathbf{v}}(\Lambda)$ of decorated representations of $\Lambda$, where $(\mathbf{d}, \mathbf{v})$ runs through $\mathbb{N}^{n} \times \mathbb{N}^{n}$. By decIrr ${ }^{\text {s.r. }}(\Lambda)$ we denote 
the subset of strongly reduced components. (The definition is in Section 5) Recall that decIrr ${ }^{\text {s.r. }}(\Lambda)$ parametrizes the elements in $\mathcal{B}_{\Lambda}$.

Let

$$
G_{\Lambda}^{\text {s.r. }}: \operatorname{dec} \operatorname{Irr}^{\text {s.r. }}(\Lambda) \rightarrow \mathbb{Z}^{n}
$$

be the map sending $Z \in \operatorname{dec} \operatorname{Irr}^{\text {s.r. }}(\Lambda)$ to the generic $g$-vector $g_{\Lambda}(Z)$ of $Z$. (The definition of a $g$-vector is in Section 3) Using Plamondon's result for finite-dimensional algebras and a long-path truncation argument, we get the following parametrization of strongly reduced components for arbitrary $\Lambda$.

Theorem 1.2. For a basic algebra $\Lambda=\mathbb{C}\langle\langle Q\rangle\rangle / I$ the following hold:

(i) The map

$$
G_{\Lambda}^{\text {s.r. }}: \operatorname{dec} \operatorname{Irr}^{\text {s.r. }}(\Lambda) \rightarrow \mathbb{Z}^{n}
$$

is injective.

(ii) The following are equivalent:

(a) $G_{\Lambda}^{\text {s.r. }}$ is surjective.

(b) $\bar{\Lambda}:=\mathbb{C}\langle\langle Q\rangle\rangle / \bar{I}$ is finite-dimensional, where $\bar{I}$ is the $\mathfrak{m}$-adic closure of $I$.

1.4. A decomposition theorem for strongly reduced components. The notion of a direct sum of irreducible components of representation varieties was introduced in CBS. The Zariski closure $Z:=\overline{Z_{1} \oplus \cdots \oplus Z_{t}}$ of a direct sum of irreducible components $Z_{1}, \ldots, Z_{t}$ of varieties of representations of $\Lambda$ is always irreducible, but in general $Z$ is not an irreducible component. It was shown in CBS that $Z$ is an irreducible component provided the dimension of the first extension group between the components is generically zero. The following decomposition theorem is an analogue for strongly reduced components. Instead of extension groups, we work with a generalization $E_{\Lambda}(-, ?)$ of the Derksen-Weyman-Zelevinsky $E$-invariant [DWZ2]. (We define $E_{\Lambda}(-, ?)$ in Section 3])

Theorem 1.3. For $Z_{1}, \ldots, Z_{t} \in \operatorname{dec} \operatorname{Irr}(\Lambda)$ the following are equivalent:

(i) $\overline{Z_{1} \oplus \cdots \oplus Z_{t}}$ is a strongly reduced irreducible component.

(ii) Each $Z_{i}$ is strongly reduced and $E_{\Lambda}\left(Z_{i}, Z_{j}\right)=0$ for all $i \neq j$.

Based on Theorem 1.3. we show that all elements of $\mathcal{B}_{\Lambda}$ can be seen as $C C$ cluster monomials. (The $C C$-cluster monomials generalize Fomin and Zelevinsky's notion of cluster monomials.)

1.5. Sign-coherence of $g$-vectors. A subset $U$ of $\mathbb{Z}^{n}$ is called sign-coherent if for each $1 \leq i \leq n$ we have either $a_{i} \geq 0$ for all $\left(a_{1}, \ldots, a_{n}\right) \in U$ or $a_{i} \leq 0$ for all $\left(a_{1}, \ldots, a_{n}\right) \in U$.

The following theorem generalizes [P2, Theorem 3.7(1)].

Theorem 1.4. Let $\Lambda$ be a basic algebra, and let $Z_{1}, \ldots, Z_{t} \in \operatorname{dec} \operatorname{Irr}^{\text {s.r. }}(\Lambda)$ be strongly reduced components. Assume that

$$
\overline{Z_{1} \oplus \cdots \oplus Z_{t}}
$$

is a strongly reduced component. Then $\left\{g_{\Lambda}\left(Z_{1}\right), \ldots, g_{\Lambda}\left(Z_{t}\right)\right\}$ is sign-coherent. 
1.6. The paper is organized as follows. In Section 2 we recall definitions and basic properties of basic algebras and their (decorated) representations. We also introduce truncations of basic algebras, which play a crucial role in some of our proofs. In Section 3 we introduce and study $g$-vectors and $E$-invariants of decorated representations. Caldero-Chapoton functions and Caldero-Chapoton algebras are defined in Section 4. Our main results, Theorems 1.2 and 1.3, are proved in Section 5. In Section 6 we introduce component graphs, component clusters and $C C$-clusters, and we show that the cardinality of loop-complete subgraphs of a component graph is bounded by the number of simple modules. Section 7 explains the relation between Caldero-Chapoton algebras and cluster algebras. Section 8 contains the proof of Theorem 1.4. Finally, in Section 9 we discuss several examples of Caldero-Chapoton algebras.

1.7. Notation. We denote the composition of maps $f: M \rightarrow N$ and $g: N \rightarrow L$ by $g f=g \circ f: M \rightarrow L$. We write $|U|$ for the cardinality of a set $U$.

A finite-dimensional module $M$ is basic provided it is a direct sum of pairwise non-isomorphic indecomposable modules. For a module $M$ and some $m \geq 1$ let $M^{m}$ be the direct sum of $m$ copies of $M$.

For a finite-dimensional algebra $\Lambda$ let $\tau_{\Lambda}$ be its Auslander-Reiten translation. For an introduction to Auslander-Reiten theory we refer to the books [ARS] and ASS.

For $n \geq 1$ and a set $S$, depending on the situation and if no misunderstanding can occur, we identify $S^{n}$ with the set of $(n \times 1)$ - or $(1 \times n)$-matrices with entries in $S$. By $\mathbb{N}$ we denote the natural numbers, including zero. For $\mathbf{d}=\left(d_{1}, \ldots, d_{n}\right) \in \mathbb{N}^{n}$ let $|\mathbf{d}|:=d_{1}+\cdots+d_{n}$. For $n \in \mathbb{N}$ let $M_{n}(\mathbb{Z})$ be the set of $(n \times n)$-matrices with integer entries.

For a ring $R$ let $R\left[x_{1}^{ \pm}, \ldots, x_{n}^{ \pm}\right]$be the algebra of Laurent polynomials over $R$ in $n$ independent variables $x_{1}, \ldots, x_{n}$. For $\mathbf{a}=\left(a_{1}, \ldots, a_{n}\right) \in \mathbb{Z}^{n}$ set $\mathbf{x}^{\mathbf{a}}:=x_{1}^{a_{1}} \cdots x_{n}^{a_{n}}$.

\section{BASIC ALGEBRAS AND DECORATED REPRESENTATIONS}

2.1. Basic algebras and quiver representations. Throughout, let $\mathbb{C}$ be the field of complex numbers. A quiver is a quadruple $Q=\left(Q_{0}, Q_{1}, s, t\right)$, where $Q_{0}$ is a finite set of vertices, $Q_{1}$ is a finite set of arrows, and $s, t: Q_{1} \rightarrow Q_{0}$ are maps. For each arrow $a \in Q_{1}$ we call $s(a)$ and $t(a)$ the starting and terminal vertex of $a$, respectively. If not mentioned otherwise, we always assume that $Q_{0}=\{1, \ldots, n\}$. Let $B_{Q}=\left(b_{i j}\right) \in M_{n}(\mathbb{Z})$, where

$$
b_{i j}:=\left|\left\{a \in Q_{1} \mid s(a)=j, t(a)=i\right\}\right|-\left|\left\{a \in Q_{1} \mid s(a)=i, t(a)=j\right\}\right| .
$$

A path in $Q$ is a tuple $p=\left(a_{m}, \ldots, a_{1}\right)$ of arrows $a_{i} \in Q_{1}$ such that $s\left(a_{i+1}\right)=$ $t\left(a_{i}\right)$ for all $1 \leq i \leq m-1$. Then $\operatorname{length}(p):=m$ is the length of $p$. Additionally, for each vertex $i \in Q_{0}$ there is a path $e_{i}$ of length 0 . We often just write $a_{m} \cdots a_{1}$ instead of $\left(a_{m}, \ldots, a_{1}\right)$.

A path $p=\left(a_{m}, \ldots, a_{1}\right)$ of length $m \geq 1$ is a cycle in $Q$, or more precisely an $m$-cycle in $Q$, if $s\left(a_{1}\right)=t\left(a_{m}\right)$. The quiver $Q$ is acyclic if there are no cycles in $Q$, and for $s \geq 1$ the quiver $Q$ is called $s$-acyclic if there are no $m$-cycles for $1 \leq m \leq s$.

A representation of a quiver $Q=\left(Q_{0}, Q_{1}, s, t\right)$ is a tuple $M=\left(M_{i}, M_{a}\right)_{i \in Q_{0}, a \in Q_{1}}$, where each $M_{i}$ is a finite-dimensional $\mathbb{C}$-vector space, and $M_{a}: M_{s(a)} \rightarrow M_{t(a)}$ is a $\mathbb{C}$-linear map for each arrow $a \in Q_{1}$. We call $\underline{\operatorname{dim}}(M):=\left(\operatorname{dim}\left(M_{1}\right), \ldots, \operatorname{dim}\left(M_{n}\right)\right)$ the dimension vector of $M$. Let $\operatorname{dim}(M):=\operatorname{dim}\left(M_{1}\right)+\cdots+\operatorname{dim}\left(M_{n}\right)$ be the 
dimension of $M$. For a path $p=\left(a_{m}, \ldots, a_{1}\right)$ in $Q$ let $M_{p}:=M_{a_{m}} \circ \cdots \circ M_{a_{1}}$. The representation $M$ is called nilpotent provided there exists some $N>0$ such that $M_{p}=0$ for all paths $p$ in $Q$ with length $(p)>N$.

For $i \in Q_{0}$ let $S_{i}:=\left(M_{i}, M_{a}\right)_{i, a}$ be the representation of $Q$ with $M_{i}=\mathbb{C}$, $M_{j}=0$ for all $j \neq i$, and $M_{a}=0$ for all $a \in Q_{1}$. For a nilpotent representation $M$ the $i$ th entry $\operatorname{dim}\left(M_{i}\right)$ of its dimension vector $\underline{\operatorname{dim}}(M)$ equals the Jordan-Hölder multiplicity $\left[M: S_{i}\right]$ of $S_{i}$ in $M$.

For $m \in \mathbb{N}$ let $\mathbb{C} Q_{m}$ be a $\mathbb{C}$-vector space with a $\mathbb{C}$-basis labeled by the set $Q_{m}$ of paths of length $m$ in $Q$. Note that $\mathbb{C} Q_{m}$ is finite-dimensional. We do not distinguish between a path $p$ of length $m$ and the corresponding basis vector in $\mathbb{C} Q_{m}$.

The completed path algebra of a quiver $Q$ is denoted by $\mathbb{C}\langle\langle Q\rangle\rangle$. As a $\mathbb{C}$-vector space we have

$$
\mathbb{C}\langle\langle Q\rangle\rangle=\prod_{m \geq 0} \mathbb{C} Q_{m}
$$

We write the elements in $\mathbb{C}\langle\langle Q\rangle\rangle$ as infinite sums $\sum_{m \geq 0} a_{m}$ with $a_{m} \in \mathbb{C} Q_{m}$. The product in $\mathbb{C}\langle\langle Q\rangle\rangle$ is then defined as

$$
\left(\sum_{i \geq 0} a_{i}\right)\left(\sum_{j \geq 0} b_{j}\right):=\sum_{k \geq 0} \sum_{i+j=k} a_{i} b_{j} .
$$

A potential of $Q$ is an element $W=\sum_{m>1} w_{m}$ of $\mathbb{C}\langle\langle Q\rangle\rangle$, where each $w_{m}$ is a $\mathbb{C}$ linear combination of $m$-cycles in $Q$. By definition, $W=0$ is also a potential. The definition of a non-degenerate potential can be found in [DWZ1, Section 7].

The category $\bmod (\mathbb{C}\langle\langle Q\rangle\rangle)$ of finite-dimensional left $\mathbb{C}\langle\langle Q\rangle\rangle$-modules can be identified with the category nil $(Q)$ of nilpotent representations of $Q$.

By $\mathfrak{m}$ we denote the arrow ideal in $\mathbb{C}\langle\langle Q\rangle\rangle$, which is generated by the arrows of $Q$. Thus for $p \geq 0$ we have

$$
\mathfrak{m}^{p}=\prod_{m \geq p} \mathbb{C} Q_{m}
$$

An ideal $I$ of $\mathbb{C}\langle\langle Q\rangle\rangle$ is admissible if $I \subseteq \mathfrak{m}^{2}$. We call an algebra $\Lambda$ basic if $\Lambda=$ $\mathbb{C}\langle\langle Q\rangle\rangle / I$ for some quiver $Q$ and some admissible ideal $I$ of $\mathbb{C}\langle\langle Q\rangle\rangle$.

A representation of a basic algebra $\Lambda=\mathbb{C}\langle\langle Q\rangle\rangle / I$ is a nilpotent representation of $Q$, which is annihilated by the ideal $I$. We identify the category $\operatorname{rep}(\Lambda)$ of representations of $\Lambda$ with the category $\bmod (\Lambda)$ of finite-dimensional left $\Lambda$-modules. Up to isomorphism the simple representations of $\Lambda$ are the 1-dimensional representations $S_{1}, \ldots, S_{n}$.

The category of all (possibly infinite-dimensional) $\Lambda$-modules is denoted by $\operatorname{Mod}(\Lambda)$. We consider $\operatorname{rep}(\Lambda)$ as a subcategory of $\operatorname{Mod}(\Lambda)$.

2.2. Decorated representations of quivers. Let $\Lambda=\mathbb{C}\langle\langle Q\rangle\rangle / I$ be a basic algebra. Following [DWZ1 (and in fact the earlier publication [MRZ]), a decorated representation of $\Lambda$ is a pair $\mathcal{M}=(M, V)$, where $M$ is a representation of $\Lambda$ and $V=\left(V_{1}, \ldots, V_{n}\right)$ is a tuple of finite-dimensional $\mathbb{C}$-vector spaces. Let $\underline{\operatorname{dim}}(V):=\left(\operatorname{dim}\left(V_{1}\right), \ldots, \operatorname{dim}\left(V_{n}\right)\right)$ and $\operatorname{dim}(V):=\operatorname{dim}\left(V_{1}\right)+\cdots+\operatorname{dim}\left(V_{n}\right)$. We call $\underline{\operatorname{dim}}(\mathcal{M}):=(\underline{\operatorname{dim}}(M), \underline{\operatorname{dim}}(V))$ the dimension vector of $\mathcal{M}$.

One defines morphisms and direct sums of decorated representations in the obvious way. Let $\operatorname{decrep}(\Lambda)$ be the category of decorated representations of $\Lambda$.

Let $\mathcal{M}=(M, V) \in \operatorname{decrep}(\Lambda)$. We write $M=0$ if all $M_{i}$ are zero, and $V=0$ if all $V_{i}$ are zero. Furthermore, $\mathcal{M}=0$ if $M=0$ and $V=0$. 
For $1 \leq i \leq n$ set $\mathcal{S}_{i}:=\left(S_{i}, 0\right)$, and let $\mathcal{S}_{i}^{-}:=(0, V)$, where $V_{i}=\mathbb{C}$ and $V_{j}=0$ for all $j \neq i$. The representations $\mathcal{S}_{i}^{-}$are the negative simple decorated representations of $\Lambda$.

2.3. Varieties of representations. For $\mathbf{d}=\left(d_{1}, \ldots, d_{n}\right) \in \mathbb{N}^{n}$ let $\operatorname{rep}_{\mathbf{d}}(\Lambda)$ be the affine variety of representations of $\Lambda$ with dimension vector d. By definition the closed points of $\operatorname{rep}_{\mathbf{d}}(\Lambda)$ are the representations $M=\left(M_{i}, M_{a}\right)_{i \in Q_{0}, a \in Q_{1}}$ of $\Lambda$ with $M_{i}=\mathbb{C}^{d_{i}}$ for all $i \in Q_{0}$. One can regard $\operatorname{rep}_{\mathbf{d}}(\Lambda)$ as a Zariski closed subset of the affine space

$$
\operatorname{rep}_{\mathbf{d}}(Q):=\prod_{a \in Q_{1}} \operatorname{Hom}_{\mathbb{C}}\left(\mathbb{C}^{d_{s(a)}}, \mathbb{C}^{d_{t(a)}}\right) .
$$

For $\mathbf{d}=\left(d_{1}, \ldots, d_{n}\right)$ let $G_{\mathbf{d}}:=\prod_{i=1}^{n} \mathrm{GL}\left(\mathbb{C}^{d_{i}}\right)$. The group $G_{\mathbf{d}}$ acts on $\operatorname{rep}_{\mathbf{d}}(\Lambda)$ by conjugation. More precisely, for $g=\left(g_{1}, \ldots, g_{n}\right) \in G_{\mathbf{d}}$ and $M \in \operatorname{rep}_{\mathbf{d}}(\Lambda)$ let

$$
g \cdot M:=\left(M_{i}, g_{t(a)}^{-1} M_{a} g_{s(a)}\right)_{i \in Q_{0}, a \in Q_{1}} .
$$

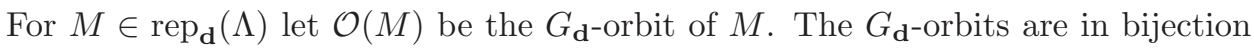
with the isomorphism classes of representations of $\Lambda$ with dimension vector $\mathbf{d}$.

For $(\mathbf{d}, \mathbf{v}) \in \mathbb{N}^{n} \times \mathbb{N}^{n}$ let $\operatorname{decrep}_{\mathbf{d}, \mathbf{v}}(\Lambda)$ be the affine variety of decorated representations $\mathcal{M}=(M, V)$ with $M \in \operatorname{rep}_{\mathbf{d}}(\Lambda)$ and $V=\mathbb{C}^{\mathbf{v}}:=\left(\mathbb{C}^{v_{1}}, \ldots, \mathbb{C}^{v_{n}}\right)$, where $\mathbf{v}=\left(v_{1}, \ldots, v_{n}\right)$.

For $\mathcal{M}=(M, V) \in \operatorname{decrep}_{\mathbf{d}, \mathbf{v}}(\Lambda)$ define $g \cdot \mathcal{M}:=(g \cdot M, V)$. This defines a $G_{\mathbf{d}^{-}}$ action on $\operatorname{decrep}_{\mathbf{d}, \mathbf{v}}(\Lambda)$. The $G_{\mathbf{d}^{-}}$orbit of $\mathcal{M}$ is denoted by $\mathcal{O}(\mathcal{M})$. We have

$$
\operatorname{dim} \mathcal{O}(\mathcal{M})=\operatorname{dim} \mathcal{O}(M)=\operatorname{dim} G_{\mathbf{d}}-\operatorname{dim} \operatorname{End}_{\Lambda}(M) ;
$$

see for example $\mathrm{G}$.

2.4. Quiver Grassmannians. Let $\Lambda=\mathbb{C}\langle\langle Q\rangle\rangle / I$ be a basic algebra. For a representation $M=\left(M_{i}, M_{a}\right)_{i \in Q_{0}, a \in Q_{1}}$ of $\Lambda$ and $\mathbf{e} \in \mathbb{N}^{n}$ let $\operatorname{Gr}_{\mathbf{e}}(M)$ be the quiver Grassmannian of subrepresentations $U$ of $M$ with $\underline{\operatorname{dim}}(U)=\mathbf{e}$. (By definition a subrepresentation of $M$ is a tuple $U=\left(U_{i}\right)_{i \in Q_{0}}$ of subspaces $U_{i} \subseteq M_{i}$ such that $M_{a}\left(U_{s(a)}\right) \subseteq U_{t(a)}$ for all $a \in Q_{1}$.) So $\operatorname{Gr}_{\mathbf{e}}(M)$ is a projective variety, which can be seen as a closed subvariety of the product of the classical Grassmannians $\operatorname{Gr}_{e_{i}}\left(M_{i}\right)$ of $e_{i}$-dimensional subspaces of $M_{i}$, where $\mathbf{e}=\left(e_{1}, \ldots, e_{n}\right)$. Let $\chi\left(\operatorname{Gr}_{\mathbf{e}}(M)\right)$ be the Euler-Poincaré characteristic of $\operatorname{Gr}_{\mathbf{e}}(M)$.

2.5. Truncations of basic algebras. For a basic algebra $\Lambda=\mathbb{C}\langle\langle Q\rangle\rangle / I$ and some $p \geq 2$ let

$$
\Lambda_{p}:=\mathbb{C}\langle\langle Q\rangle\rangle /\left(I+\mathfrak{m}^{p}\right)
$$

be the $p$-truncation of $\Lambda$. Clearly, $\Lambda_{p}$ is a finite-dimensional basic algebra. Let

$$
\bar{\Lambda}:=\mathbb{C}\langle\langle Q\rangle\rangle / \bar{I}
$$

where

$$
\bar{I}:=\bigcap_{p \geq 0}\left(I+\mathfrak{m}^{p}\right)
$$

is the $\mathfrak{m}$-adic closure of $I$ in $\mathbb{C}\langle\langle Q\rangle\rangle$. We obtain the following commutative diagram with exact rows, where all morphisms whose label contains the symbol $\iota$ (resp. $\pi$ ) 
are the obvious canonical monomorphisms (resp. epimorphisms):

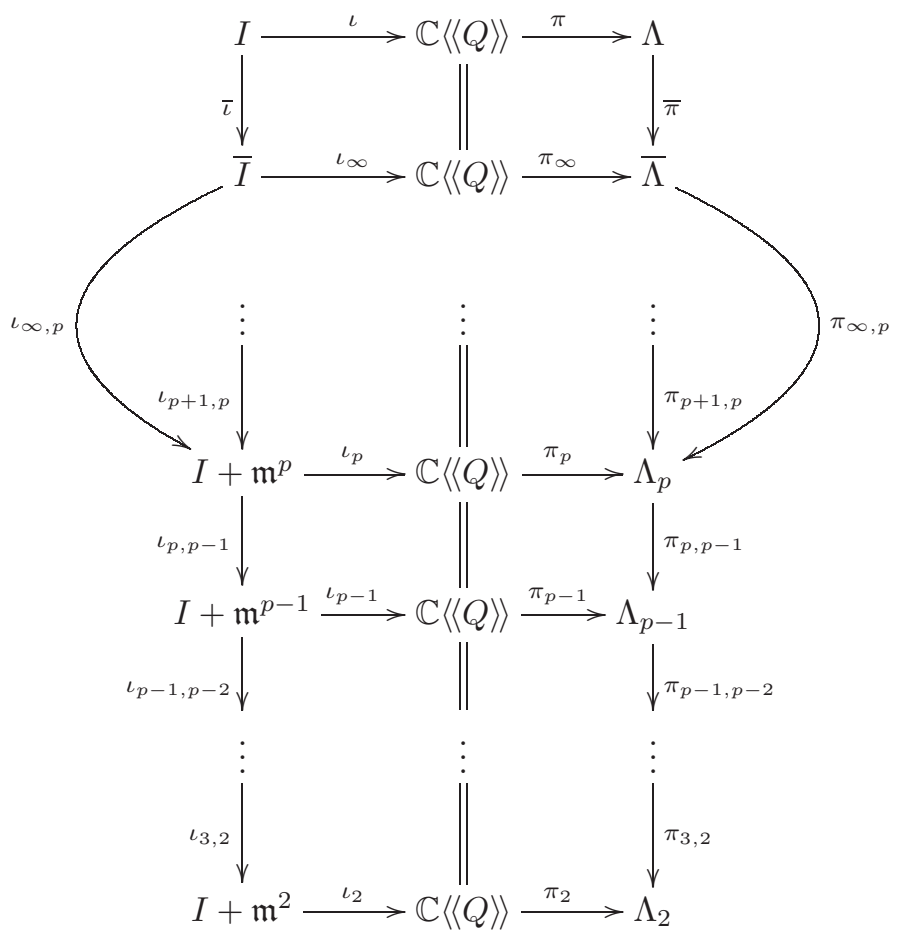

We have

$$
\bar{\Lambda}=\lim _{\longleftarrow}\left(\Lambda_{p}\right)
$$

i.e. the algebra $\bar{\Lambda}$ is the inverse limit of the inverse system

$$
\left(\cdots \rightarrow \Lambda_{p} \rightarrow \cdots \rightarrow \Lambda_{3} \rightarrow \Lambda_{2}\right) .
$$

The epimorphisms in the third column of the above diagram induce sequences

$$
\operatorname{rep}\left(\Lambda_{2}\right) \rightarrow \cdots \rightarrow \operatorname{rep}\left(\Lambda_{p}\right) \rightarrow \cdots \rightarrow \operatorname{rep}(\bar{\Lambda}) \rightarrow \operatorname{rep}(\Lambda)
$$

and

$$
\operatorname{decrep}\left(\Lambda_{2}\right) \rightarrow \cdots \rightarrow \operatorname{decrep}\left(\Lambda_{p}\right) \rightarrow \cdots \rightarrow \operatorname{decrep}(\bar{\Lambda}) \rightarrow \operatorname{decrep}(\Lambda)
$$

of embedding functors. We can consider these as chains of subcategories of $\operatorname{rep}(\Lambda)$ and $\operatorname{decrep}(\Lambda)$, respectively. The following lemma is straightforward.

Lemma 2.1. For any basic algebra $\Lambda$ we have

$$
\operatorname{rep}(\Lambda)=\operatorname{rep}(\bar{\Lambda})=\bigcup_{p \geq 2} \operatorname{rep}\left(\Lambda_{p}\right) \quad \text { and } \quad \operatorname{decrep}(\Lambda)=\operatorname{decrep}(\bar{\Lambda})=\bigcup_{p \geq 2} \operatorname{decrep}\left(\Lambda_{p}\right) .
$$

For $1 \leq i \leq n$ and $p \geq 2$ let $I_{i, p} \in \operatorname{rep}\left(\Lambda_{p}\right), \bar{I}_{i} \in \operatorname{Mod}(\bar{\Lambda})$ and $I_{i} \in \operatorname{Mod}(\Lambda)$ be the injective envelopes of the simple module $S_{i}$. The above embedding functors yield a chain

$$
I_{i, 2} \subseteq I_{i, 3} \subseteq \cdots \subseteq I_{i, p} \subseteq \cdots \subseteq \bar{I}_{i}
$$

of submodules of $\bar{I}_{i}$, and we have

$$
\bar{I}_{i}=\bigcup_{p \geq 2} I_{i, p}
$$


Lemma 2.2. Let $\Lambda=\mathbb{C}\langle\langle Q\rangle\rangle / I$ be a basic algebra. Then the following hold:

(i) Let $\mathcal{M}=(M, V) \in \operatorname{decrep}(\Lambda)$. If $p \geq \operatorname{dim}(M)$, then $\mathcal{M}$ is in the image of the embedding decrep $\left(\Lambda_{p}\right) \rightarrow \operatorname{decrep}(\Lambda)$.

(ii) Let $M, N \in \operatorname{rep}(\Lambda)$. If $p \geq \operatorname{dim}(M), \operatorname{dim}(N)$, then

$$
\operatorname{dim} \operatorname{Hom}_{\Lambda_{p}}(M, N)=\operatorname{dim} \operatorname{Hom}_{\Lambda}(M, N) .
$$

(iii) Let $M, N \in \operatorname{rep}(\Lambda)$. If $p \geq \operatorname{dim}(M)+\operatorname{dim}(N)$, then

$$
\operatorname{dim} \operatorname{Ext}_{\Lambda_{p}}^{1}(M, N)=\operatorname{dim} \operatorname{Ext}_{\Lambda}^{1}(M, N) .
$$

(iv) Let $(\mathbf{d}, \mathbf{v}) \in \mathbb{N}^{n} \times \mathbb{N}^{n}$. If $p \geq|\mathbf{d}|$, then $\operatorname{decrep}_{\mathbf{d}, \mathbf{v}}\left(\Lambda_{p}\right)=\operatorname{decrep}_{\mathbf{d}, \mathbf{v}}(\Lambda)$.

Proof. Let $a_{m} \cdots a_{1}$ be a path of length $m$ in $Q$, and let $M$ be a representation of $\Lambda$. We can see $M$ as a nilpotent representation of $Q$. For any non-zero vector $v_{0} \in M$ set $v_{i}:=a_{i} \cdots a_{1} v_{0}$ for $1 \leq i \leq m$. Assume that each of the vectors $v_{1}, \ldots, v_{m}$ is non-zero. We claim that $v_{0}, v_{1}, \ldots, v_{m}$ are pairwise different and linearly independent. Let $b$ be a path of maximal length such that $b v_{0} \neq 0$. Such a path $b$ exists, because $M$ is nilpotent. By induction $v_{1}, \ldots, v_{m}$ are linearly independent. Assume now that

$$
v_{0}=\sum_{i=1}^{m} \lambda_{i} v_{i}
$$

for some $\lambda_{i} \in \mathbb{C}$. We have $v_{i}=a_{i} \cdots a_{1} v_{0}$. Therefore we get

$$
b v_{0}=\sum_{i=1}^{m} \lambda_{i} b a_{i} \cdots a_{1} v_{0} .
$$

Since $b a_{i} \cdots a_{1}$ is either zero or a path of length $i+\operatorname{length}(b)$, we have $b a_{i} \cdots a_{1} v_{0}=0$ for all $1 \leq i \leq m$. Since $b v_{0} \neq 0$, this is a contradiction. Therefore $v_{0}, v_{1}, \ldots, v_{m}$ are linearly independent. It follows that for any $\mathcal{M} \in \operatorname{decrep}(\Lambda)$ with $\underline{\operatorname{dim}}(\mathcal{M})=(\mathbf{d}, \mathbf{v})$ and any path $b$ with length $(b) \geq|\mathbf{d}|$ we have $b \mathcal{M}=0$. This implies (i). Parts (ii) and (iv) are easy consequences of (i). Any extension of representations $M$ and $N$ of $\Lambda$ is a representation of $\Lambda$ of dimension $\operatorname{dim}(M)+\operatorname{dim}(N)$. This implies (iii).

\section{E-INVARIANTS AND $g$-VECTORS OF DECORATED REPRESENTATIONS}

3.1. Definition of $E$-invariants and $g$-vectors. Let $Q$ be a quiver, and let $W$ be a potential of $Q$. Let $\Lambda=\mathcal{P}(Q, W)$ be the associated Jacobian algebra [DWZ1, Section 3]. For decorated representations $\mathcal{M}$ and $\mathcal{N}$ of $\Lambda$ the $g$-vector $g(\mathcal{M})$ and the invariants $E^{\text {inj }}(\mathcal{M})$ and $E^{\text {inj }}(\mathcal{M}, \mathcal{N})$ were defined in DWZ2, where $E^{\text {inj }}(\mathcal{M})$ is called the $E$-invariant of $\mathcal{M}$. We define invariants $g_{\Lambda}(\mathcal{M}), E_{\Lambda}(\mathcal{M})$ and $E_{\Lambda}(\mathcal{M}, \mathcal{N})$ of decorated representations $\mathcal{M}$ and $\mathcal{N}$ of an arbitrary basic algebra $\Lambda=\mathbb{C}\langle\langle Q\rangle\rangle / I$ as follows.

For a decorated representation $\mathcal{M}=(M, V)$ of $\Lambda$ let

$$
g_{\Lambda}(\mathcal{M}):=\left(g_{1}, \ldots, g_{n}\right)
$$

with

$$
g_{i}:=g_{i}(\mathcal{M}):=-\operatorname{dim} \operatorname{Hom}_{\Lambda}\left(S_{i}, M\right)+\operatorname{dim} \operatorname{Ext}_{\Lambda}^{1}\left(S_{i}, M\right)+\operatorname{dim}\left(V_{i}\right)
$$

be the $g$-vector of $\mathcal{M}$. 
For decorated representations $\mathcal{M}=(M, V)$ and $\mathcal{N}=(N, W)$ of $\Lambda$ let

$$
E_{\Lambda}(\mathcal{M}, \mathcal{N}):=\operatorname{dim} \operatorname{Hom}_{\Lambda}(M, N)+\sum_{i=1}^{n} \operatorname{dim}\left(M_{i}\right) g_{i}(\mathcal{N})
$$

The E-invariant of $\mathcal{M}$ is defined as $E_{\Lambda}(\mathcal{M}):=E_{\Lambda}(\mathcal{M}, \mathcal{M})$.

Lemma 3.1. Let $\Lambda=\mathcal{P}(Q, W)$, where $W$ is a potential of $Q$. For $\mathcal{M}, \mathcal{N} \in$ $\operatorname{decrep}(\Lambda)$ the following hold:

(i) $g_{\Lambda}(\mathcal{M})=g(\mathcal{M})$.

(ii) $E_{\Lambda}(\mathcal{M}, \mathcal{N})=E^{\operatorname{inj}}(\mathcal{M}, \mathcal{N})$.

Proof. Part (i) follows from [P1, Lemma 4.7, Proposition 4.8]. It can also be shown in a more elementary way by using the exact sequence displayed in DWZ2, Equation (10.4)]. Part (ii) is a direct consequence of (i) and the definition of $E_{\Lambda}(\mathcal{M}, \mathcal{N})$ and $E^{\operatorname{inj}}(\mathcal{M}, \mathcal{N})$.

3.2. Homological interpretation of the $E$-invariant. For $1 \leq i \leq n$ let $I_{i} \in$ $\operatorname{Mod}(\Lambda)$ be the injective envelope of the simple representation $S_{i}$ of $\Lambda$. One easily checks that the socle $\operatorname{soc}\left(I_{i}\right)$ of $I_{i}$ is isomorphic to $S_{i}$ and that

$$
\operatorname{dim} \operatorname{Hom}_{\Lambda}\left(M, I_{i}\right)=\operatorname{dim}\left(M_{i}\right)
$$

for all $M \in \operatorname{rep}(\Lambda)$. Note that in general $I_{i}$ is infinite dimensional. For $M \in \operatorname{rep}(\Lambda)$ let

$$
0 \rightarrow M \stackrel{f}{\rightarrow} I_{0}^{\Lambda}(M) \rightarrow I_{1}^{\Lambda}(M)
$$

denote a minimal injective presentation of $M$. The modules $I_{0}^{\Lambda}(M)$ and $I_{1}^{\Lambda}(M)$ are up to isomorphism uniquely determined by $M$.

We will need the following theorem due to Auslander and Reiten.

Theorem 3.2 ([AR, Theorem $1.4(\mathrm{~b})]$ ). Let $M$ and $N$ be representations of a finite-dimensional basic algebra $\Lambda$. Then we have

$$
\begin{aligned}
\operatorname{dim} \operatorname{Hom}_{\Lambda}\left(\tau_{\Lambda}^{-}(N), M\right)=\operatorname{dim} \operatorname{Hom}_{\Lambda}(M, N)-\operatorname{dim} \operatorname{Hom}_{\Lambda}\left(M, I_{0}^{\Lambda}(N)\right) \\
+\operatorname{dim}_{\operatorname{Hom}_{\Lambda}\left(M, I_{1}^{\Lambda}(N)\right) .}
\end{aligned}
$$

Lemma 3.3. Let $\Lambda=\mathbb{C}\langle\langle Q\rangle\rangle / I$ be a finite-dimensional basic algebra, and let $M \in$ $\operatorname{rep}(\Lambda)$. Let

$$
0 \rightarrow M \stackrel{f}{\rightarrow} I_{0}^{\Lambda}(M) \rightarrow I_{1}^{\Lambda}(M)
$$

be a minimal injective presentation of $M$. Then for $1 \leq i \leq n$ we have

(i) $\left[\operatorname{soc}\left(I_{0}^{\Lambda}(M)\right): S_{i}\right]=\left[\operatorname{soc}(M): S_{i}\right]=\operatorname{dim} \operatorname{Hom}_{\Lambda}\left(S_{i}, M\right)$ and

$$
I_{0}^{\Lambda}(M) \cong I_{1}^{\operatorname{dim} \operatorname{Hom}_{\Lambda}\left(S_{1}, M\right)} \oplus \cdots \oplus I_{n}^{\operatorname{dim} \operatorname{Hom}_{\Lambda}\left(S_{n}, M\right)} .
$$

(ii) $\left[\operatorname{soc}\left(I_{1}^{\Lambda}(M)\right): S_{i}\right]=\left[\operatorname{soc}(\operatorname{Coker}(f)): S_{i}\right]=\operatorname{dim}_{\operatorname{Ext}_{\Lambda}^{1}\left(S_{i}, M\right) \text { and }}$

$$
I_{1}^{\Lambda}(M) \cong I_{1}^{\operatorname{dim} \operatorname{Ext}_{\Lambda}^{1}\left(S_{1}, M\right)} \oplus \cdots \oplus I_{n}^{\operatorname{dim} \operatorname{Ext}_{\Lambda}^{1}\left(S_{n}, M\right)} .
$$

Proof. Since $I_{0}^{\Lambda}(M)$ is the injective envelope of $M$, we have $\operatorname{soc}(M) \cong \operatorname{soc}\left(I_{0}^{\Lambda}(M)\right)$. This implies (i). By the construction of minimal injective presentations, $I_{1}^{\Lambda}(M)$ is the injective envelope of $\operatorname{Coker}(f)$. It follows that $\operatorname{soc}(\operatorname{Coker}(f)) \cong \operatorname{soc}\left(I_{1}^{\Lambda}(M)\right)$. We apply the functor $\operatorname{Hom}_{\Lambda}\left(S_{i},-\right)$ to the exact sequence

$$
0 \rightarrow M \stackrel{f}{\rightarrow} I_{0}^{\Lambda}(M) \rightarrow \operatorname{Coker}(f) \rightarrow 0 .
$$


This yields an exact sequence

$$
\begin{aligned}
0 & \rightarrow \operatorname{Hom}_{\Lambda}\left(S_{i}, M\right) \stackrel{F}{\rightarrow} \operatorname{Hom}_{\Lambda}\left(S_{i}, I_{0}^{\Lambda}(M)\right) \\
& \rightarrow \operatorname{Hom}_{\Lambda}\left(S_{i}, \operatorname{Coker}(f)\right) \stackrel{G}{\rightarrow} \operatorname{Ext}_{\Lambda}^{1}\left(S_{i}, M\right) \rightarrow 0 .
\end{aligned}
$$

Here we used that $I_{0}^{\Lambda}(M)$ is injective, which implies $\operatorname{Ext}_{\Lambda}^{1}\left(S_{i}, I_{0}^{\Lambda}(M)\right)=0$. By (i) we know that $F$ is an isomorphism. Thus $G$ is also an isomorphism. This implies (ii).

Combining Lemma 2.2 and Lemma 3.3 yields the following result.

Lemma 3.4. Let $\mathcal{M}=(M, V)$ be a decorated representation of a basic algebra $\Lambda$, and let $g_{\Lambda}(\mathcal{M})=\left(g_{1}, \ldots, g_{n}\right)$ be the $g$-vector of $\mathcal{M}$. If $p>\operatorname{dim}(M)$, then

$$
g_{i}=-\left[\operatorname{soc}\left(I_{0}^{\Lambda_{p}}(M)\right): S_{i}\right]+\left[\operatorname{soc}\left(I_{1}^{\Lambda_{p}}(M)\right): S_{i}\right]+\operatorname{dim}\left(V_{i}\right)
$$

for all $1 \leq i \leq n$.

The following result is a homological interpretation of the $E$-invariant in terms of Auslander-Reiten translations. This can be seen as a generalization of [DWZ2, Corollary 10.9].

Proposition 3.5. Let $\mathcal{M}=(M, V)$ and $\mathcal{N}=(N, W)$ be decorated representations of a basic algebra $\Lambda$. If $p>\operatorname{dim}(M), \operatorname{dim}(N)$, then

$$
E_{\Lambda}(\mathcal{M}, \mathcal{N})=E_{\Lambda_{p}}(\mathcal{M}, \mathcal{N})=\operatorname{dim} \operatorname{Hom}_{\Lambda_{p}}\left(\tau_{\Lambda_{p}}^{-}(N), M\right)+\sum_{i=1}^{n} \operatorname{dim}\left(M_{i}\right) \operatorname{dim}\left(W_{i}\right)
$$

In particular, we have

$$
E_{\Lambda_{p}}(\mathcal{M}, \mathcal{N})=E_{\Lambda_{q}}(\mathcal{M}, \mathcal{N})
$$

and

$$
\operatorname{dim} \operatorname{Hom}_{\Lambda_{p}}\left(\tau_{\Lambda_{p}}^{-}(N), M\right)=\operatorname{dim} \operatorname{Hom}_{\Lambda_{q}}\left(\tau_{\Lambda_{q}}^{-}(N), M\right)
$$

for all $p, q>\operatorname{dim}(M), \operatorname{dim}(N)$.

Proof. Since $p>\operatorname{dim}(M), \operatorname{dim}(N)$ we can apply Lemma 2.2 and get

$$
\begin{aligned}
\operatorname{dim} \operatorname{Hom}_{\Lambda_{p}}(M, N) & =\operatorname{dim} \operatorname{Hom}_{\Lambda}(M, N), \\
\operatorname{dim} \operatorname{Hom}_{\Lambda_{p}}\left(S_{i}, N\right) & =\operatorname{dim} \operatorname{Hom}_{\Lambda}\left(S_{i}, N\right), \\
\operatorname{dim} \operatorname{Ext}_{\Lambda_{p}}^{1}\left(S_{i}, N\right) & =\operatorname{dim} \operatorname{Ext}_{\Lambda}^{1}\left(S_{i}, N\right) .
\end{aligned}
$$

Let

$$
0 \rightarrow N \rightarrow I_{0}^{\Lambda_{p}}(N) \rightarrow I_{1}^{\Lambda_{p}}(N)
$$

be a minimal injective presentation of $N$, where we regard $N$ now as a representation of $\Lambda_{p}$. It follows from Lemma 3.3 and equation (2) that

$$
\begin{aligned}
& \operatorname{dim} \operatorname{Hom}_{\Lambda_{p}}\left(M, I_{0}^{\Lambda_{p}}(N)\right)=\sum_{i=1}^{n} \operatorname{dim}\left(M_{i}\right) \operatorname{dim} \operatorname{Hom}_{\Lambda_{p}}\left(S_{i}, N\right), \\
& \operatorname{dim} \operatorname{Hom}_{\Lambda_{p}}\left(M, I_{1}^{\Lambda_{p}}(N)\right)=\sum_{i=1}^{n} \operatorname{dim}\left(M_{i}\right) \operatorname{dim} \operatorname{Ext}_{\Lambda_{p}}^{1}\left(S_{i}, N\right) .
\end{aligned}
$$


This implies

$$
\begin{aligned}
& E_{\Lambda}(\mathcal{M}, \mathcal{N})=\operatorname{dim} \operatorname{Hom}_{\Lambda}(M, N)+\sum_{i=1}^{n} \operatorname{dim}\left(M_{i}\right)\left(-\operatorname{dim} \operatorname{Hom}_{\Lambda}\left(S_{i}, N\right)\right. \\
& \left.+\operatorname{dim} \operatorname{Ext}_{\Lambda}^{1}\left(S_{i}, N\right)\right)+\sum_{i=1}^{n} \operatorname{dim}\left(M_{i}\right) \operatorname{dim}\left(W_{i}\right) \\
& =\operatorname{dim} \operatorname{Hom}_{\Lambda_{p}}(M, N)+\sum_{i=1}^{n} \operatorname{dim}\left(M_{i}\right)\left(-\operatorname{dim} \operatorname{Hom}_{\Lambda_{p}}\left(S_{i}, N\right)\right. \\
& \left.+\operatorname{dim} \operatorname{Ext}_{\Lambda_{p}}^{1}\left(S_{i}, N\right)\right)+\sum_{i=1}^{n} \operatorname{dim}\left(M_{i}\right) \operatorname{dim}\left(W_{i}\right) \\
& =\operatorname{dim} \operatorname{Hom}_{\Lambda_{p}}(M, N)-\operatorname{dim} \operatorname{Hom}_{\Lambda_{p}}\left(M, I_{0}^{\Lambda_{p}}(N)\right) \\
& +\operatorname{dim} \operatorname{Hom}_{\Lambda_{p}}\left(M, I_{1}^{\Lambda_{p}}(N)\right)+\sum_{i=1}^{n} \operatorname{dim}\left(M_{i}\right) \operatorname{dim}\left(W_{i}\right) .
\end{aligned}
$$

The first equality follows from Lemmas $2.2,3.3$ and 3.4. The second equality says that $E_{\Lambda}(\mathcal{M}, \mathcal{N})=E_{\Lambda_{p}}(\mathcal{M}, \mathcal{N})$. Applying Theorem 3.2 yields

$$
E_{\Lambda_{p}}(\mathcal{M}, \mathcal{N})=\operatorname{dim} \operatorname{Hom}_{\Lambda_{p}}\left(\tau_{\Lambda_{p}}^{-}(N), M\right)+\sum_{i=1}^{n} \operatorname{dim}\left(M_{i}\right) \operatorname{dim}\left(W_{i}\right) .
$$

This finishes the proof.

Corollary 3.6. For decorated representations $\mathcal{M}$ and $\mathcal{N}$ of a basic algebra $\Lambda$ we have

$$
E_{\Lambda}(\mathcal{M}, \mathcal{N}) \geq 0
$$

\section{Caldero-Chapoton algebras}

4.1. Caldero-Chapoton functions. To any basic algebra $\Lambda=\mathbb{C}\langle\langle Q\rangle\rangle / I$ we associate a set of Laurent polynomials in $n$ independent variables $x_{1}, \ldots, x_{n}$ as follows. The Caldero-Chapoton function associated to a decorated representation $\mathcal{M}=(M, V)$ of $\Lambda$ is defined as

$$
C_{\Lambda}(\mathcal{M}):=\mathbf{x}^{g_{\Lambda}(\mathcal{M})} \sum_{\mathbf{e} \in \mathbb{N}^{n}} \chi\left(\operatorname{Gr}_{\mathbf{e}}(M)\right) \mathbf{x}^{B_{Q} \mathbf{e}},
$$

where $B_{Q}$ and $g_{\Lambda}(\mathcal{M})$ are defined as in Sections 2.1 and 3.1 respectively. Note that $C_{\Lambda}(\mathcal{M}) \in \mathbb{Z}\left[x_{1}^{ \pm}, \ldots, x_{n}^{ \pm}\right]$. Let

$$
\mathcal{C}_{\Lambda}:=\left\{C_{\Lambda}(\mathcal{M}) \mid \mathcal{M} \in \operatorname{decrep}(\Lambda)\right\}
$$

be the set of Caldero-Chapoton functions associated to $\Lambda$. For $\mathcal{M}=(M, 0)$ we sometimes write $C_{\Lambda}(M)$ instead of $C_{\Lambda}(\mathcal{M})$.

The definition of $C_{\Lambda}(\mathcal{M})$ is motivated by the (different versions of) CalderoChapoton functions appearing in the theory of cluster algebras; see for example [Pa, Section 1]. Such functions first appeared in work of Caldero and Chapoton [CC, Section 3], where they show that the cluster variables of a cluster algebra of a Dynkin quiver are Caldero-Chapoton functions. 
Lemma 4.1. For decorated representations $\mathcal{M}=(M, V)$ and $\mathcal{N}=(N, W)$ the following hold:

(i) $g_{\Lambda}(\mathcal{M} \oplus \mathcal{N})=g_{\Lambda}(\mathcal{M})+g_{\Lambda}(\mathcal{N})$.

(ii) $C_{\Lambda}(\mathcal{M})=C_{\Lambda}(M, 0) C_{\Lambda}(0, V)$.

(iii) $C_{\Lambda}(\mathcal{M} \oplus \mathcal{N})=C_{\Lambda}(\mathcal{M}) C_{\Lambda}(\mathcal{N})$.

Proof. Part (i) follows directly from the definitions and from the additivity of the functors $\operatorname{Hom}_{\Lambda}(-, ?)$ and $\operatorname{Ext}_{\Lambda}^{1}(-, ?)$. To prove (ii), let $\mathcal{M}=(M, V)$ be a decorated representation of $\Lambda$. For the decorated representation $(0, V)$ we have

$$
C_{\Lambda}(0, V)=\prod_{i=1}^{n} x_{i}^{v_{i}}
$$

where $\underline{\operatorname{dim}}(V)=\left(v_{1}, \ldots, v_{n}\right)$. For the decorated representation $(M, 0)$ we have

$$
C_{\Lambda}(M, 0):=\mathbf{x}^{g_{\Lambda}(M, 0)} \sum_{\mathbf{e} \in \mathbb{N}^{n}} \chi\left(\operatorname{Gr}_{\mathbf{e}}(M)\right) \mathbf{x}^{B_{Q} \mathbf{e}}
$$

where $g_{i}(M, 0)=-\operatorname{dim} \operatorname{Hom}_{\Lambda}\left(S_{i}, M\right)+\operatorname{dim}_{\operatorname{Ext}_{\Lambda}^{1}}\left(S_{i}, M\right)$ for $1 \leq i \leq n$. Now one easily checks that $C_{\Lambda}(\mathcal{M})=C_{\Lambda}(M, 0) C_{\Lambda}(0, V)$. Thus (ii) holds. Now (iii) follows from (i), (ii) and the well known formula

$$
\chi\left(\operatorname{Gr}_{\mathbf{e}}(M \oplus N)\right)=\sum_{\left(\mathbf{e}^{\prime}, \mathbf{e}^{\prime \prime}\right)} \chi\left(\operatorname{Gr}_{\mathbf{e}^{\prime}}(M)\right) \chi\left(\operatorname{Gr}_{\mathbf{e}^{\prime \prime}}(N)\right)
$$

where the sum runs over all pairs $\left(\mathbf{e}^{\prime}, \mathbf{e}^{\prime \prime}\right) \in \mathbb{N}^{n} \times \mathbb{N}^{n}$ such that $\mathbf{e}^{\prime}+\mathbf{e}^{\prime \prime}=\mathbf{e}$; see for example [DWZ2, Proof of Proposition 3.2].

4.2. Definition of a Caldero-Chapoton algebra. In the previous section, we associated to a basic algebra $\Lambda$ the set

$$
\mathcal{C}_{\Lambda}=\left\{C_{\Lambda}(\mathcal{M}) \mid \mathcal{M} \in \operatorname{decrep}(\Lambda)\right\}
$$

of Caldero-Chapoton functions. Clearly, $\mathcal{C}_{\Lambda}$ is a subset of the integer Laurent polynomial ring $\mathbb{Z}\left[x_{1}^{ \pm}, \ldots, x_{n}^{ \pm}\right]$generated by the variables $x_{1}, \ldots, x_{n}$. By definition the Caldero-Chapoton algebra $\mathcal{A}_{\Lambda}$ associated to $\Lambda$ is the $\mathbb{C}$-subalgebra of $\mathbb{C}\left[x_{1}^{ \pm}, \ldots, x_{n}^{ \pm}\right]$ generated by $\mathcal{C}_{\Lambda}$. The following is a direct consequence of Lemma 4.1(iii).

Lemma 4.2. The set $\mathcal{C}_{\Lambda}$ generates $\mathcal{A}_{\Lambda}$ as a $\mathbb{C}$-vector space.

In this generality, Caldero-Chapoton algebras might not be so useful. (One could generalize even more by replacing the matrix $B_{Q}$ in the definition of the functions $C_{\Lambda}(\mathcal{M})$ by any other matrix in $M_{n}(\mathbb{Z})$.) But the case where $\Lambda$ is the Jacobian algebra $\mathcal{P}(Q, W)$ of a quiver $Q$ with non-degenerate potential $W$ (see DWZ1 for missing definitions) should certainly be of interest. In this case, based on work of Palu $\mathrm{Pa}$, Plamondon [P1] considered a version of Caldero-Chapoton functions using the Amiot cluster category [A]. In contrast, we follow Derksen, Weyman and Zelevinsky's DWZ1, DWZ2 approach and define and study CalderoChapoton functions purely in terms of the representation theory of the Jacobian algebra without passing to the cluster category. 
4.3. Linear independence of Caldero-Chapoton functions. Let $\Lambda=\mathbb{C}\langle\langle Q\rangle\rangle / I$ be a basic algebra. Except in some trivial cases, the set $\mathcal{C}_{\Lambda}$ of Caldero-Chapoton functions associated to decorated representations of $\Lambda$ is linearly dependent. Often the Caldero-Chapoton functions satisfy beautiful relations, which should be studied more intensively. On the other hand, by Lemma 4.2. there are $\mathbb{C}$-bases of $\mathcal{A}_{\Lambda}$ consisting only of Caldero-Chapoton functions. Our aim is to provide a candidate $\mathcal{B}_{\Lambda}$ for such a basis. Before constructing $\mathcal{B}_{\Lambda}$ in Section 5 we prove the following criterion for linear independence of certain sets of Caldero-Chapoton functions.

Let

$$
\begin{aligned}
& \mathbb{Q}_{\geq 0}^{n}:=\left\{\left(a_{1}, \ldots, a_{n}\right) \in \mathbb{Q}^{n} \mid a_{i} \geq 0 \text { for all } i\right\}, \\
& \mathbb{Q}_{>0}^{n}:=\left\{\left(a_{1}, \ldots, a_{n}\right) \in \mathbb{Q}^{n} \mid a_{i}>0 \text { for all } i\right\} .
\end{aligned}
$$

Proposition 4.3. Let $\Lambda=\mathbb{C}\langle\langle Q\rangle\rangle / I$ be a basic algebra. Let $\mathcal{M}_{j}, j \in J$ be decorated representations of $\Lambda$. Assume the following:

(i) $\operatorname{Ker}\left(B_{Q}\right) \cap \mathbb{Q}_{\geq 0}^{n}=0$.

(ii) The $g$-vectors $g_{\Lambda}\left(\mathcal{M}_{j}\right), j \in J$ are pairwise different.

Then the Caldero-Chapoton functions $C_{\Lambda}\left(\mathcal{M}_{j}\right), j \in J$ are pairwise different and linearly independent in $\mathcal{A}_{\Lambda}$.

Proof. We treat $B_{Q}$ as a linear map $\mathbb{Q}^{n} \rightarrow \mathbb{Q}^{n}$. For $\mathbf{a}, \mathbf{b} \in \mathbb{Z}^{n}$ define $\mathbf{a} \leq \mathbf{b}$ if there exists some $\mathbf{e} \in \mathbb{Q}_{\geq 0}^{n}$ such that

$$
\mathbf{a}=\mathbf{b}+B_{Q} \mathbf{e}
$$

We claim that this defines a partial order on $\mathbb{Z}^{n}$. Clearly, $\leq$ is reflexive and transitive. Assume that $\mathbf{a} \leq \mathbf{b}$ and $\mathbf{b} \leq \mathbf{a}$. Thus $\mathbf{a}=\mathbf{b}+B_{Q} \mathbf{f}_{1}$ and $\mathbf{b}=\mathbf{a}+B_{Q} \mathbf{f}_{2}$ for some $\mathbf{f}_{1}, \mathbf{f}_{2} \in \mathbb{Q}_{\geq 0}^{n}$. It follows that $\mathbf{a}=\mathbf{a}+B_{Q}\left(\mathbf{f}_{1}+\mathbf{f}_{2}\right)$. Thus $\mathbf{f}_{1}+\mathbf{f}_{2} \in \operatorname{Ker}\left(B_{Q}\right)$. Our assumption (i) yields that $\mathbf{f}_{1}=\mathbf{f}_{2}=0$. Thus $\mathbf{a}=\mathbf{b}$. This shows that $\leq$ is antisymmetric.

The partial order $\leq$ on $\mathbb{Z}^{n}$ induces obviously a partial order on the set of Laurent monomials in the variables $x_{1}, \ldots, x_{n}$. Namely, set $\mathbf{x}^{\mathbf{a}} \leq \mathbf{x}^{\mathbf{b}}$ if $\mathbf{a} \leq \mathbf{b}$. Let $\operatorname{deg}\left(\mathbf{x}^{\mathbf{a}}\right):=\mathbf{a}$ be the degree of $\mathbf{x}^{\mathbf{a}}$.

Among the Laurent monomials $\mathbf{x}^{g_{\Lambda}(\mathcal{M})+B_{Q} \mathbf{e}}$ occurring in the expression

$$
C_{\Lambda}(\mathcal{M})=\mathbf{x}^{g_{\Lambda}(\mathcal{M})} \sum_{\mathbf{e} \in \mathbb{N}^{n}} \chi\left(\operatorname{Gr}_{\mathbf{e}}(M)\right) \mathbf{x}^{B_{Q} \mathbf{e}}=\sum_{\mathbf{e} \in \mathbb{N}^{n}} \chi\left(\operatorname{Gr}_{\mathbf{e}}(M)\right) \mathbf{x}^{g_{\Lambda}(\mathcal{M})+B_{Q} \mathbf{e}}
$$

the monomial $\mathbf{x}^{g_{\Lambda}(\mathcal{M})}$ is the unique monomial of maximal degree.

For $\mathbf{e}=0$ the Grassmannian $\operatorname{Gr}_{\mathbf{e}}(M)$ is just a point, and $B_{Q} \mathbf{e}=0$. Thus, if $\mathbf{e}=0$, we have $\chi\left(\operatorname{Gr}_{\mathbf{e}}(M)\right) \mathbf{x}^{B_{Q} \mathbf{e}}=1$. This shows that the Laurent monomial $\mathbf{x}^{g_{\Lambda}(\mathcal{M})}$ really occurs as a non-trivial summand of $C_{\Lambda}(\mathcal{M})$. In particular, we have $C_{\Lambda}(\mathcal{M}) \neq C_{\Lambda}(\mathcal{N})$ if $g_{\Lambda}(\mathcal{M}) \neq g_{\Lambda}(\mathcal{N})$.

Now let $\mathcal{M}_{1}, \ldots, \mathcal{M}_{t}$ be decorated representations of $\Lambda$ with pairwise different $g$-vectors. Assume that

$$
\lambda_{1} C_{\Lambda}\left(\mathcal{M}_{1}\right)+\cdots+\lambda_{t} C_{\Lambda}\left(\mathcal{M}_{t}\right)=0
$$

for some $\lambda_{j} \in \mathbb{C}$. Without loss of generality we assume that $\lambda_{j} \neq 0$ for all $j$. There is a (not necessarily unique) index $s$ such that $\mathrm{x}^{g_{\Lambda}\left(\mathcal{M}_{s}\right)}$ is maximal in the set $\left\{\mathbf{x}^{g_{\Lambda}\left(\mathcal{M}_{j}\right)} \mid 1 \leq j \leq t\right\}$. It follows that the Laurent monomial $\mathbf{x}^{g_{\Lambda}\left(\mathcal{M}_{s}\right)}$ does not occur as a summand of any of the Laurent polynomials $C_{\Lambda}\left(\mathcal{M}_{j}\right)$ with $j \neq s$. 
(Here we use that the $g$-vectors of the decorated representations $\mathcal{M}_{j}$ are pairwise different.) This implies $\lambda_{s}=0$, a contradiction. Thus $C_{\Lambda}\left(\mathcal{M}_{1}\right), \ldots, C_{\Lambda}\left(\mathcal{M}_{t}\right)$ are linearly independent.

For $\mathbf{a}=\left(a_{1}, \ldots, a_{n}\right)$ and $\mathbf{b}=\left(b_{1}, \ldots, b_{n}\right)$ in $\mathbb{Z}^{n}$ set $\mathbf{a} \cdot \mathbf{b}:=a_{1} b_{1}+\cdots+a_{n} b_{n}$.

Note that condition (d) in the following lemma coincides with condition (i) in Proposition 4.3

Lemma 4.4. For the conditions

(a) $\operatorname{rank}\left(B_{Q}\right)=n$;

(b) each row of $B_{Q}$ has at least one non-zero entry, and there are $n-\operatorname{rank}\left(B_{Q}\right)$ rows of $B_{Q}$, which are non-negative linear combinations of the remaining $\operatorname{rank}\left(B_{Q}\right)$ rows of $B_{Q}$;

(c) $\operatorname{Im}\left(B_{Q}\right) \cap \mathbb{Q}_{>0}^{n} \neq \varnothing$;

(d) $\operatorname{Ker}\left(B_{Q}\right) \cap \mathbb{Q}_{\geq 0}^{n}=0$,

the implications

$$
(\mathrm{a}) \Longrightarrow(\mathrm{b}) \Longrightarrow(\mathrm{c}) \Longrightarrow(\mathrm{d})
$$

hold.

Proof. The implication (a) $\Longrightarrow$ (b) is trivial. Next, assume (b) holds. Let $m:=\operatorname{rank}\left(B_{Q}\right)$. We denote the $j$ th row of $B_{Q}$ by $r_{j}$. By assumption there are pairwise different indices $i_{1}, \ldots, i_{m} \in\{1, \ldots, n\}$ such that for each $1 \leq k \leq n$ with $k \notin\left\{i_{1}, \ldots, i_{m}\right\}$ we have

$$
r_{k}=\lambda_{1}^{(k)} r_{i_{1}}+\cdots+\lambda_{m}^{(k)} r_{i_{m}}
$$

for some non-negative rational numbers $\lambda_{j}^{(k)}$. Since $r_{k}$ is non-zero, at least one of the $\lambda_{j}^{(k)}$ is positive. Clearly, there is some $\mathbf{e} \in \mathbb{Q}^{n}$ such that $r_{i_{j}} \cdot \mathbf{e}=1$ for all $1 \leq j \leq m$. (The $(k \times n)$-matrix with rows $r_{i_{1}}, \ldots, r_{i_{m}}$ has rank $m$. Thus, we can see it as a surjective homomorphism $\mathbb{Q}^{n} \rightarrow \mathbb{Q}^{m}$.) Now observe that the $k$ th entry of $B_{Q} \mathbf{e}$ is $\lambda_{1}^{(k)}+\cdots+\lambda_{m}^{(k)}$ for all $1 \leq k \leq n$ with $k \notin\left\{i_{1}, \ldots, i_{m}\right\}$ and that this entry is positive. It follows that $\operatorname{Im}\left(B_{Q}\right) \cap \mathbb{Q}_{>0}^{n} \neq \varnothing$.

Finally, to show (c) $\Longrightarrow$ (d) let $\mathbf{b} \in \operatorname{Im}\left(B_{Q}\right) \cap \mathbb{Q}_{>0}^{n}$. Thus there is some $\mathbf{a} \in \mathbb{Q}^{n}$ such that $B_{Q} \mathbf{a}=\mathbf{b}$. Since $B_{Q}$ is skew-symmetric, we get $-\mathbf{a} B_{Q}=\mathbf{b}$. Now let $\mathbf{e} \in \operatorname{Ker}\left(B_{Q}\right) \cap \mathbb{Q}_{\geq 0}^{n}$. We get $B_{Q} \mathbf{e}=0$, and therefore $-\mathbf{a} B_{Q} \mathbf{e}=\mathbf{b} \cdot \mathbf{e}=0$. Since $\mathbf{b}$ has only positive entries and $\mathbf{e}$ has only non-negative entries, we get $\mathbf{e}=0$. This finishes the proof.

For the example, where $\Lambda$ is the path algebra of an affine quiver of type $\mathbb{A}_{2}$, the main argument used in the proof of Proposition 4.3 can already be found in [C, Section 6.1]. If we replace condition (i) by condition (a), Proposition 4.3 was first proved by $\mathrm{Fu}$ and Keller [FK, Corollary 4.4]. Essentially the same argument was later also used by Plamondon [P1]. That the Fu-Keller argument can be applied under condition (b) was observed by Geiß and Labardini. To any triangulation $T$ of a punctured Riemann surface with non-empty boundary, one can associate a 2-acyclic quiver $Q_{T}$. It is shown in GLaS that there is always a triangulation $T$ such that the matrix $B_{Q_{T}}$ satisfies condition (b). 


\section{Strongly Reduced Components of RePRESEntation VARIETIES}

5.1. Strongly reduced components. Let $\Lambda=\mathbb{C}\langle\langle Q\rangle\rangle / I$ be a basic algebra, and let $(\mathbf{d}, \mathbf{v}) \in \mathbb{N}^{n} \times \mathbb{N}^{n}$. By $\operatorname{Irr}_{\mathbf{d}}(\Lambda)$ and $\operatorname{dec}_{\operatorname{Irr}} \mathbf{d , \mathbf { v }}(\Lambda)$ we denote the set of irreducible components of $\operatorname{rep}_{\mathbf{d}}(\Lambda)$ and $\operatorname{decrep}_{\mathbf{d}, \mathbf{v}}(\Lambda)$, respectively. For $Z \in \operatorname{dec}_{\operatorname{Irr}} \mathbf{d , \mathbf { v }}(\Lambda)$ we write $\underline{\operatorname{dim}}(Z):=(\mathbf{d}, \mathbf{v})$. Let

$$
\operatorname{Irr}(\Lambda)=\bigcup_{\mathbf{d} \in \mathbb{N}^{n}} \operatorname{Irr}_{\mathbf{d}}(\Lambda) \text { and } \operatorname{dec} \operatorname{Irr}(\Lambda)=\bigcup_{(\mathbf{d}, \mathbf{v}) \in \mathbb{N}^{n} \times \mathbb{N}^{n}} \operatorname{dec}_{\operatorname{Irr}} \mathbf{d}, \mathbf{v}(\Lambda) .
$$

Note that any irreducible component $Z \in \operatorname{dec} \operatorname{Irr}(\Lambda)$ can be seen as an irreducible component in $\operatorname{Irr}\left(\Lambda_{\mathrm{dec}}\right)$, where $\Lambda_{\mathrm{dec}}:=\Lambda \times \mathbb{C} \times \cdots \times \mathbb{C}$ is defined as the product of $\Lambda$ with $n$ copies of $\mathbb{C}$. In fact, we can identify $\operatorname{decrep}(\Lambda)$ and $\operatorname{rep}\left(\Lambda_{\text {dec }}\right)$. Thus statements on varieties of representations can be carried over to varieties of decorated representations.

By definition we have

$$
\operatorname{decrep}_{\mathbf{d}, \mathbf{v}}(\Lambda)=\left\{\left(M, \mathbb{C}^{\mathbf{v}}\right) \mid M \in \operatorname{rep}_{\mathbf{d}}(\Lambda)\right\} .
$$

We have an isomorphism

$$
\operatorname{decrep}_{\mathbf{d}, \mathbf{v}}(\Lambda) \rightarrow \operatorname{rep}_{\mathbf{d}}(\Lambda)
$$

of affine varieties mapping $\left(M, \mathbb{C}^{\mathbf{v}}\right)$ to $M$. Thus the irreducible components of $\operatorname{decrep}_{\mathbf{d}, \mathbf{v}}(\Lambda)$ can be interpreted as irreducible components of $\operatorname{rep}_{\mathbf{d}}(\Lambda)$. For $Z \in$ $\operatorname{dec}_{\operatorname{Irr}_{\mathbf{d}, \mathbf{v}}}(\Lambda)$ let $\pi Z$ be the corresponding component in $\operatorname{Irr}_{\mathbf{d}}(\Lambda)$.

For $Z, Z_{1}, Z_{2} \in \operatorname{dec} \operatorname{Irr}(\Lambda)$ define

$$
\begin{aligned}
c_{\Lambda}(Z) & :=\min \{\operatorname{dim}(Z)-\operatorname{dim} \mathcal{O}(\mathcal{M}) \mid \mathcal{M} \in Z\}, \\
e_{\Lambda}(Z) & :=\min \left\{\operatorname{dim} \operatorname{Ext}_{\Lambda}^{1}(M, M) \mid \mathcal{M}=(M, V) \in Z\right\}, \\
\operatorname{end}_{\Lambda}(Z) & :=\min \left\{\operatorname{dim}_{\left.\operatorname{End}_{\Lambda}(M) \mid \mathcal{M}=(M, V) \in Z\right\},}\right. \\
\operatorname{hom}_{\Lambda}\left(Z_{1}, Z_{2}\right) & :=\min \left\{\operatorname{dim} \operatorname{Hom}_{\Lambda}\left(M_{1}, M_{2}\right) \mid \mathcal{M}_{i}=\left(M_{i}, V_{i}\right) \in Z_{i}, i=1,2\right\}, \\
\operatorname{ext}_{\Lambda}^{1}\left(Z_{1}, Z_{2}\right) & :=\min \left\{\operatorname{dim} \operatorname{Ext}_{\Lambda}^{1}\left(M_{1}, M_{2}\right) \mid \mathcal{M}_{i}=\left(M_{i}, V_{i}\right) \in Z_{i}, i=1,2\right\} .
\end{aligned}
$$

For $Z, Z_{1}, Z_{2} \in \operatorname{dec} \operatorname{Irr}(\Lambda)$ there is a dense open subset $U$ of $Z$ (resp. $Z_{1} \times Z_{2}$ ) such that $E_{\Lambda}(\mathcal{M})=E_{\Lambda}(\mathcal{N})$ for all $\mathcal{M}, \mathcal{N} \in U$ (resp. $E_{\Lambda}\left(\mathcal{M}_{1}, \mathcal{M}_{2}\right)=E_{\Lambda}\left(\mathcal{N}_{1}, \mathcal{N}_{2}\right)$ for all $\left.\left(\mathcal{M}_{1}, \mathcal{M}_{2}\right),\left(\mathcal{N}_{1}, \mathcal{N}_{2}\right) \in U\right)$. This follows from the upper semicontinuity of the functions $\operatorname{dim} \operatorname{Hom}_{\Lambda}\left(-\right.$, ?) and $\operatorname{dim} \operatorname{Ext}_{\Lambda}^{1}(-, ?)$ proved in [CBS, Lemma 4.3]. For $\mathcal{M} \in U$ (resp. $\left.\left(\mathcal{M}_{1}, \mathcal{M}_{2}\right) \in U\right)$ define $E_{\Lambda}(Z):=E_{\Lambda}(\mathcal{M})\left(\right.$ resp. $E_{\Lambda}\left(Z_{1}, Z_{2}\right):=$ $\left.E_{\Lambda}\left(\mathcal{M}_{1}, \mathcal{M}_{2}\right)\right)$.

Note that for $Z \in \operatorname{dec}_{\operatorname{Irr}_{\mathbf{d}, \mathbf{v}}}(\Lambda)$ we have

$$
c_{\Lambda}(Z)=\operatorname{dim}(Z)-\operatorname{dim}\left(G_{\mathbf{d}}\right)+\operatorname{end}_{\Lambda}(Z) .
$$

This follows from equation (10).

It is easy to construct examples of components $Z \in \operatorname{dec} \operatorname{Irr}(\Lambda)$ such that $\operatorname{end}_{\Lambda}(Z)$ $\neq \operatorname{hom}_{\Lambda}(Z, Z), e_{\Lambda}(Z) \neq \operatorname{ext}_{\Lambda}^{1}(Z, Z)$ and $E_{\Lambda}(Z) \neq E_{\Lambda}(Z, Z)$. Namely, let $\Lambda=\mathbb{C} Q$ be the path algebra of the Kronecker quiver, and let $Z \in \operatorname{dec}_{\operatorname{Irr}} \mathbf{d}, \mathbf{v}(\Lambda)$ with $\mathbf{d}=$ $(1,1)$ and $\mathbf{v}=(0,0)$. (Since $\Lambda$ is a path algebra of an acyclic quiver, $\operatorname{decrep}_{\mathbf{d}, \mathbf{v}}(\Lambda)$ is irreducible for all $\mathbf{d}, \mathbf{v}$.) An easy calculation shows that $\operatorname{end}_{\Lambda}(Z)=e_{\Lambda}(Z)=$ $E_{\Lambda}(Z)=1$ and $\operatorname{hom}_{\Lambda}(Z, Z)=\operatorname{ext}_{\Lambda}^{1}(Z, Z)=E_{\Lambda}(Z, Z)=0$. A further discussion of this example can be found in Section 9.4 .3 .

The next lemma follows again from the upper semicontinuity of $\operatorname{dim} \operatorname{Hom}_{\Lambda}(-, ?)$ and $\operatorname{dim} \operatorname{Ext}_{\Lambda}^{1}(-, ?)$. 
Lemma 5.1. For $Z, Z_{1}, Z_{2} \in \operatorname{dec} \operatorname{Irr}(\Lambda)$ the following hold:

(i) The sets

$$
\begin{aligned}
& \left\{\mathcal{M} \in Z \mid \operatorname{dim}(Z)-\operatorname{dim} \mathcal{O}(\mathcal{M})=c_{\Lambda}(Z)\right\}, \\
& \left\{\mathcal{M}=(M, V) \in Z \mid \operatorname{dim}_{\operatorname{Ext}_{\Lambda}^{1}}(M, M)=e_{\Lambda}(Z)\right\} \text {, } \\
& \left\{\mathcal{M}=(M, V) \in Z \mid \operatorname{dim}_{\operatorname{End}_{\Lambda}}(M)=\operatorname{end}_{\Lambda}(Z)\right\}
\end{aligned}
$$

are open in $Z$.

(ii) The sets

$$
\begin{aligned}
& \left\{\left(\left(M_{1}, V_{1}\right),\left(M_{2}, V_{2}\right)\right) \in Z_{1} \times Z_{2} \mid \operatorname{dim} \operatorname{Hom}_{\Lambda}\left(M_{1}, M_{2}\right)=\operatorname{hom}_{\Lambda}\left(Z_{1}, Z_{2}\right)\right\}, \\
& \left\{\left(\left(M_{1}, V_{1}\right),\left(M_{2}, V_{2}\right)\right) \in Z_{1} \times Z_{2} \mid \operatorname{dim} \operatorname{Ext}_{\Lambda}^{1}\left(M_{1}, M_{2}\right)=\operatorname{ext}_{\Lambda}^{1}\left(Z_{1}, Z_{2}\right)\right\} \\
& \quad \text { are open in } Z_{1} \times Z_{2} .
\end{aligned}
$$

(iii) The sets

$$
\begin{aligned}
& \left\{\mathcal{M} \in Z \mid E_{\Lambda}(\mathcal{M})=E_{\Lambda}(Z)\right\} \\
& \left\{\left(\mathcal{M}_{1}, \mathcal{M}_{2}\right) \in Z_{1} \times Z_{2} \mid E_{\Lambda}\left(\mathcal{M}_{1}, \mathcal{M}_{2}\right)=E_{\Lambda}\left(Z_{1}, Z_{2}\right)\right\}
\end{aligned}
$$

are dense constructible subsets of $Z$ and $Z_{1} \times Z_{2}$, respectively.

Lemma 5.2. For $Z, Z_{1}, Z_{2} \in \operatorname{dec} \operatorname{Irr}(\Lambda)$ we have

$$
c_{\Lambda}(Z) \leq e_{\Lambda}(Z) \leq E_{\Lambda}(Z) \quad \text { and } \quad \operatorname{ext}_{\Lambda}^{1}\left(Z_{1}, Z_{2}\right) \leq E_{\Lambda}\left(Z_{1}, Z_{2}\right) .
$$

Proof. Let $\mathbf{d}=\underline{\operatorname{dim}}(\pi Z)$ and $\mathbf{d}_{i}=\underline{\operatorname{dim}}\left(\pi Z_{i}\right)$ for $i=1,2$. Choose some $p \geq$ $2|\mathbf{d}|,\left|\mathbf{d}_{1}\right|+\left|\mathbf{d}_{2}\right|$. By Lemma 2.2 we can regard all the representations in $Z, Z_{1}$ and $Z_{2}$ as representations of $\Lambda_{p}$. Thus we can interpret $Z, Z_{1}$ and $Z_{2}$ as irreducible components in $\operatorname{dec} \operatorname{Irr}\left(\Lambda_{p}\right)$. Now Proposition 3.5 allows us to assume without loss of generality that $\Lambda=\Lambda_{p}$. Voigt's Lemma [G. Proposition 1.1] implies that $c_{\Lambda}(Z) \leq$ $e_{\Lambda}(Z)$. The Auslander-Reiten formula $\operatorname{Ext}_{\Lambda}^{1}(M, N) \cong \operatorname{DHom}_{\Lambda}\left(\tau_{\Lambda}^{-}(N), M\right)$ yields

$$
\operatorname{dim} \operatorname{Ext}_{\Lambda}^{1}(M, N) \leq \operatorname{dim} \operatorname{Hom}_{\Lambda}\left(\tau_{\Lambda}^{-}(N), M\right) .
$$

This implies $e_{\Lambda}(Z) \leq E_{\Lambda}(Z)$ and $\operatorname{ext}_{\Lambda}^{1}\left(Z_{1}, Z_{2}\right) \leq E_{\Lambda}\left(Z_{1}, Z_{2}\right)$. (Here we used again Proposition 3.5.)

Following GLS we call an irreducible component $Z \in \operatorname{dec} \operatorname{Irr}(\Lambda)$ strongly reduced provided

$$
c_{\Lambda}(Z)=e_{\Lambda}(Z)=E_{\Lambda}(Z) .
$$

For example, if $\Lambda$ is finite-dimensional, one can easily check that for any injective $\Lambda$ module $I \in \operatorname{rep}(\Lambda)$ the closure of the orbit $\mathcal{O}(I, 0)$ is a strongly reduced irreducible component. Similarly, it follows directly from the definitions that for all decorated representations of the form $\mathcal{M}=(0, V)$, the closure of $\mathcal{O}(\mathcal{M})$ is a strongly reduced component. (In this case, $\mathcal{O}(\mathcal{M})$ is just a point, and it is equal to its closure.)

Let $\operatorname{dec}_{\operatorname{Irr}}^{\text {s.r. }} \mathbf{\text { s. }}(\Lambda)$ be the set of all strongly reduced components of $\operatorname{decrep}_{\mathbf{d}, \mathbf{v}}(\Lambda)$, and let

$$
\operatorname{dec} \operatorname{Irr}^{\text {s.r. }}(\Lambda):=\bigcup_{(\mathbf{d}, \mathbf{v}) \in \mathbb{N}^{n} \times \mathbb{N}^{n}} \operatorname{dec}_{\operatorname{Irr}}^{\text {s.r. }} \mathbf{d}, \mathbf{v}(\Lambda)
$$


5.2. Decomposition theorems for irreducible components. An irreducible component $Z$ in $\operatorname{Irr}(\Lambda)$ or $\operatorname{dec} \operatorname{Irr}(\Lambda)$ is called indecomposable provided there exists a dense open subset $U$ of $Z$ which contains only indecomposable representations

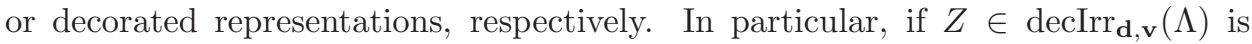
indecomposable, then either $\mathbf{d}=0$ or $\mathbf{v}=0$.

Given irreducible components $Z_{i}$ of $\operatorname{decrep}_{\mathbf{d}_{i}, \mathbf{v}_{i}}(\Lambda)$ for $1 \leq i \leq t$, let $(\mathbf{d}, \mathbf{v}):=$ $\left(\mathbf{d}_{1}, \mathbf{v}_{1}\right)+\cdots+\left(\mathbf{d}_{t}, \mathbf{v}_{t}\right)$ and let

$$
Z_{1} \oplus \cdots \oplus Z_{t}
$$

be the points of $\operatorname{decrep}_{\mathbf{d}, \mathbf{v}}(\Lambda)$ which are isomorphic to $M_{1} \oplus \cdots \oplus M_{t}$ with $M_{i} \in Z_{i}$ for $1 \leq i \leq t$. The Zariski closure of $Z_{1} \oplus \cdots \oplus Z_{t}$ in $\operatorname{decrep}_{\mathbf{d}, \mathbf{v}}(\Lambda)$ is denoted by

$$
\overline{Z_{1} \oplus \cdots \oplus Z_{t}} \text {. }
$$

It is quite easy to show that $\overline{Z_{1} \oplus \cdots \oplus Z_{t}}$ is an irreducible closed subset of decrep $_{\mathbf{d}, \mathbf{v}}(\Lambda)$, but in general it is not an irreducible component.

Theorem 5.3 (CBS). For $Z_{1}, \ldots, Z_{t} \in \operatorname{dec} \operatorname{Irr}(\Lambda)$ the following are equivalent:

(i) $\overline{Z_{1} \oplus \cdots \oplus Z_{t}}$ is an irreducible component.

(ii) $\operatorname{ext}_{\Lambda}^{1}\left(Z_{i}, Z_{j}\right)=0$ for all $i \neq j$.

Furthermore, the following hold:

(iii) Each irreducible component $Z \in \operatorname{dec} \operatorname{Irr}(\Lambda)$ can be written as $Z=$ $\overline{Z_{1} \oplus \cdots \oplus Z_{t}}$ with $Z_{1}, \ldots, Z_{t}$ indecomposable irreducible components in $\operatorname{dec} \operatorname{Irr}(\Lambda)$. Suppose that

$$
\overline{Z_{1} \oplus \cdots \oplus Z_{t}}=\overline{Z_{1}^{\prime} \oplus \cdots \oplus Z_{s}^{\prime}}
$$

is an irreducible component with $Z_{i}$ and $Z_{i}^{\prime}$ indecomposable irreducible components in $\operatorname{dec} \operatorname{Irr}(\Lambda)$ for all $i$. Then $s=t$ and there is a bijection $\sigma:\{1, \ldots, t\}$ $\rightarrow\{1, \ldots, s\}$ such that $Z_{i}=Z_{\sigma(i)}^{\prime}$ for all $i$.

The next lemma is an easy exercise.

Lemma 5.4. For $1 \leq i \leq n$ and any decorated representation $\mathcal{M}=(M, V)$ of $\Lambda$ we have

$$
E_{\Lambda}\left(\mathcal{M}, \mathcal{S}_{i}^{-}\right)=\operatorname{dim}\left(M_{i}\right) \quad \text { and } \quad E_{\Lambda}\left(\mathcal{S}_{i}^{-}, \mathcal{M}\right)=0 .
$$

Corollary 5.5. If $\operatorname{dec}_{\operatorname{Irr}}^{\text {s.r. }} \mathbf{v}(\Lambda) \neq \varnothing$, then we have $d_{i} v_{i}=0$ for all $1 \leq i \leq n$.

Proof. Let $Z \in \operatorname{dec}_{\operatorname{Irr}}^{\text {s.r. }} \mathbf{d}(\Lambda)$ for some $\mathbf{d}, \mathbf{v}$, and let $Z^{\prime}$ be the corresponding irreducible component of $\operatorname{dec}_{\operatorname{Irr}} \mathbf{d}, 0(\Lambda)$. We clearly have $c_{\Lambda}(Z)=c_{\Lambda}\left(Z^{\prime}\right)$ and $E_{\Lambda}(Z)=$ $E_{\Lambda}\left(Z^{\prime}\right)+d_{1} v_{1}+\cdots+d_{n} v_{n}$. Using Lemma 5.2 we obtain $c_{\Lambda}(Z)=c_{\Lambda}\left(Z^{\prime}\right) \leq E_{\Lambda}\left(Z^{\prime}\right) \leq$ $E_{\Lambda}(Z)$. Since $c_{\Lambda}(Z)=E_{\Lambda}(Z)$, the result follows.

Lemma 5.6. Let $Z \in \operatorname{dec}_{\operatorname{Irr}} \mathbf{d , \mathbf { v }}(\Lambda)$, and assume that $p>|\mathbf{d}|$. Then the following are equivalent:

(i) $Z \in \operatorname{dec} \operatorname{Irr}^{\text {s.r. }}(\Lambda)$.

(ii) $Z \in \operatorname{dec} \operatorname{Irr}^{\text {s.r. }}\left(\Lambda_{p}\right)$.

Proof. Since $p>|\mathbf{d}|$, we can apply Lemma2.2 and Proposition 3.5 and get $c_{\Lambda_{p}}(Z)=$ $c_{\Lambda}(Z)$ and $E_{\Lambda_{p}}(Z)=E_{\Lambda}(Z)$. This yields the result.

The additivity of the functor $\operatorname{Hom}_{\Lambda}(-, ?)$ and upper semicontinuity imply the following lemma. 
Lemma 5.7. Let $Z, Z_{1}, Z_{2} \in \operatorname{dec} \operatorname{Irr}(\Lambda)$. Suppose that $Z=\overline{Z_{1} \oplus Z_{2}}$. Then the following hold:

(i) $\operatorname{end}_{\Lambda}(Z)=\operatorname{end}_{\Lambda}\left(Z_{1}\right)+\operatorname{end}_{\Lambda}\left(Z_{2}\right)+\operatorname{hom}_{\Lambda}\left(Z_{1}, Z_{2}\right)+\operatorname{hom}_{\Lambda}\left(Z_{2}, Z_{1}\right)$.

(ii) $E_{\Lambda}(Z)=E_{\Lambda}\left(Z_{1}\right)+E_{\Lambda}\left(Z_{2}\right)+E_{\Lambda}\left(Z_{1}, Z_{2}\right)+E_{\Lambda}\left(Z_{2}, Z_{1}\right)$.

Recall that for $\mathbf{a}=\left(a_{1}, \ldots, a_{n}\right)$ and $\mathbf{b}=\left(b_{1}, \ldots, b_{n}\right)$ in $\mathbb{Z}^{n}$ we defined $\mathbf{a} \cdot \mathbf{b}:=$ $a_{1} b_{1}+\cdots+a_{n} b_{n}$. The following lemma is obvious.

Lemma 5.8. Let $\mathbf{d}, \mathbf{d}_{1}, \mathbf{d}_{2} \in \mathbb{N}^{n}$ with $\mathbf{d}=\mathbf{d}_{1}+\mathbf{d}_{2}$. Then

$$
\operatorname{dim}\left(G_{\mathbf{d}}\right)-\operatorname{dim}\left(G_{\mathbf{d}_{1}}\right)-\operatorname{dim}\left(G_{\mathbf{d}_{2}}\right)=2\left(\mathbf{d}_{1} \cdot \mathbf{d}_{2}\right) .
$$

Lemma 5.9. Let $Z, Z_{1}, Z_{2} \in \operatorname{dec} \operatorname{Irr}(\Lambda)$ with $Z=\overline{Z_{1} \oplus Z_{2}}$. We have

$$
\begin{aligned}
\operatorname{dim}(Z)= & \operatorname{dim}\left(Z_{1}\right)+\operatorname{dim}\left(Z_{2}\right)+2\left(\underline{\operatorname{dim}}\left(\pi Z_{1}\right) \cdot \underline{\operatorname{dim}}\left(\pi Z_{2}\right)\right) \\
& -\operatorname{hom}_{\Lambda}\left(Z_{1}, Z_{2}\right)-\operatorname{hom}_{\Lambda}\left(Z_{2}, Z_{1}\right) .
\end{aligned}
$$

Proof. For $i=1,2$ let $\left(\mathbf{d}_{i}, \mathbf{v}_{i}\right):=\underline{\operatorname{dim}}\left(Z_{i}\right)$, and let $(\mathbf{d}, \mathbf{v}):=\underline{\operatorname{dim}}(Z)$. We have $\underline{\operatorname{dim}}(Z)=\underline{\operatorname{dim}}\left(Z_{1}\right)+\underline{\operatorname{dim}}\left(Z_{2}\right)$ and $\underline{\operatorname{dim}}\left(\pi Z_{i}\right)=\mathbf{d}_{i}$. The map

$$
f: G_{\mathbf{d}} \times Z_{1} \times Z_{2} \rightarrow Z
$$

defined by

$$
\left(g,\left(M_{1}, \mathbb{C}^{\mathbf{v}_{1}}\right),\left(M_{2}, \mathbb{C}^{\mathbf{v}_{2}}\right)\right) \mapsto\left(g \cdot\left(M_{1} \oplus M_{2}\right), \mathbb{C}^{\mathbf{v}}\right)
$$

is a morphism of affine varieties. For $\left(\mathcal{M}_{1}, \mathcal{M}_{2}\right) \in Z_{1} \times Z_{2}$ define

$$
f_{\mathcal{M}_{1}, \mathcal{M}_{2}}: G_{\mathbf{d}} \times \mathcal{O}\left(\mathcal{M}_{1}\right) \times \mathcal{O}\left(\mathcal{M}_{2}\right) \rightarrow \mathcal{O}\left(\mathcal{M}_{1} \oplus \mathcal{M}_{2}\right)
$$

by $\left(g, \mathcal{N}_{1}, \mathcal{N}_{2}\right) \mapsto f\left(g, \mathcal{N}_{1}, \mathcal{N}_{2}\right)$. The fibres of $f_{\mathcal{M}_{1}, \mathcal{M}_{2}}$ are of dimension

$$
d_{\mathcal{M}_{1}, \mathcal{M}_{2}}:=\operatorname{dim}\left(G_{\mathbf{d}}\right)+\operatorname{dim} \mathcal{O}\left(\mathcal{M}_{1}\right)+\operatorname{dim} \mathcal{O}\left(\mathcal{M}_{2}\right)-\operatorname{dim} \mathcal{O}\left(\mathcal{M}_{1} \oplus \mathcal{M}_{2}\right) .
$$

Using equation (11), an easy calculation yields

$$
d_{\mathcal{M}_{1}, \mathcal{M}_{2}}=\operatorname{dim}\left(G_{\mathbf{d}_{1}}\right)+\operatorname{dim}\left(G_{\mathbf{d}_{2}}\right)+\operatorname{dim} \operatorname{Hom}_{\Lambda}\left(M_{1}, M_{2}\right)+\operatorname{dim} \operatorname{Hom}_{\Lambda}\left(M_{2}, M_{1}\right) .
$$

Let $\mathcal{M}$ be in the image of $f$. We want to compute the dimension of the fibre $f^{-1}(\mathcal{M})$. Let

$$
\mathcal{T}:=\left\{\mathcal{O}\left(\mathcal{N}_{1}\right) \times \mathcal{O}\left(\mathcal{N}_{2}\right) \subseteq Z_{1} \times Z_{2} \mid \mathcal{N}_{1} \oplus \mathcal{N}_{2} \cong \mathcal{M}\right\}
$$

It follows from the Krull-Remak-Schmidt Theorem that $\mathcal{T}$ is a finite set. Thus the fibre $f^{-1}(\mathcal{M})$ is the disjoint union of the fibres $f_{\mathcal{N}_{1}, \mathcal{N}_{2}}^{-1}(\mathcal{M})$, where $\mathcal{O}\left(\mathcal{N}_{1}\right) \times \mathcal{O}\left(\mathcal{N}_{2}\right)$ runs through $\mathcal{T}$. So we get

$$
\operatorname{dim}\left(f^{-1}(\mathcal{M})\right)=\max \left\{d_{\mathcal{N}_{1}, \mathcal{N}_{2}} \mid \mathcal{O}\left(\mathcal{N}_{1}\right) \times \mathcal{O}\left(\mathcal{N}_{2}\right) \in \mathcal{T}\right\} .
$$

Thus by upper semicontinuity there is a dense open subset $V \subseteq Z$ such that all fibres $f^{-1}(\mathcal{M})$ with $\mathcal{M} \in V$ have dimension

$$
d_{Z_{1}, Z_{2}}:=\operatorname{dim}\left(G_{\mathbf{d}_{1}}\right)+\operatorname{dim}\left(G_{\mathbf{d}_{2}}\right)+\operatorname{hom}_{\Lambda}\left(Z_{1}, Z_{2}\right)+\operatorname{hom}_{\Lambda}\left(Z_{2}, Z_{1}\right) .
$$

We have

$$
\operatorname{dim}(Z)+d_{Z_{1}, Z_{2}}=\operatorname{dim}\left(G_{\mathbf{d}}\right)+\operatorname{dim}\left(Z_{1}\right)+\operatorname{dim}\left(Z_{2}\right) .
$$

Using Lemma 5.8 we get

$$
\operatorname{dim}(Z)=\operatorname{dim}\left(Z_{1}\right)+\operatorname{dim}\left(Z_{2}\right)+2\left(\mathbf{d}_{1} \cdot \mathbf{d}_{2}\right)-\operatorname{hom}_{\Lambda}\left(Z_{1}, Z_{2}\right)-\operatorname{hom}_{\Lambda}\left(Z_{2}, Z_{1}\right) .
$$

This finishes the proof. 
Lemma 5.10. For $Z, Z_{1}, Z_{2} \in \operatorname{dec} \operatorname{Irr}(\Lambda)$ with $Z=\overline{Z_{1} \oplus Z_{2}}$ we have

$$
c_{\Lambda}(Z)=c_{\Lambda}\left(Z_{1}\right)+c_{\Lambda}\left(Z_{2}\right) .
$$

Proof. For $i=1,2$ let $\left(\mathbf{d}_{i}, \mathbf{v}_{i}\right):=\underline{\operatorname{dim}}\left(Z_{i}\right)$, and let $(\mathbf{d}, \mathbf{v}):=\underline{\operatorname{dim}}(Z)$. We get

$$
\begin{aligned}
c_{\Lambda}(Z) & =\operatorname{dim}(Z)-\operatorname{dim}\left(G_{\mathbf{d}}\right)+\operatorname{end}_{\Lambda}(Z) \\
& =\operatorname{dim}\left(Z_{1}\right)+\operatorname{dim}\left(Z_{2}\right)-\operatorname{dim}\left(G_{\mathbf{d}_{1}}\right)-\operatorname{dim}\left(G_{\mathbf{d}_{2}}\right)+\operatorname{end}_{\Lambda}\left(Z_{1}\right)+\operatorname{end}_{\Lambda}\left(Z_{2}\right) \\
& =c_{\Lambda}\left(Z_{1}\right)+c_{\Lambda}\left(Z_{2}\right) .
\end{aligned}
$$

The first equality follows directly from the definition of $c_{\Lambda}(Z)$. The second equality uses Lemma 5.7(i) and Lemma 5.9.

The following result is a version of Theorem 5.3 for strongly reduced components.

Theorem 5.11. For $Z_{1}, \ldots, Z_{t} \in \operatorname{dec} \operatorname{Irr}(\Lambda)$ the following are equivalent:

(i) $\overline{Z_{1} \oplus \cdots \oplus Z_{t}}$ is a strongly reduced irreducible component.

(ii) Each $Z_{i}$ is strongly reduced and $E_{\Lambda}\left(Z_{i}, Z_{j}\right)=0$ for all $i \neq j$.

Proof. Without loss of generality assume that $t=2$. The general case follows by induction. Let $Z_{1} \in \operatorname{dec} \operatorname{Irr}_{\mathbf{d}_{1}, \mathbf{v}_{1}}(\Lambda)$ and $Z_{2} \in \operatorname{dec}_{\operatorname{Irr}_{\mathbf{d}_{2}, \mathbf{v}_{2}}}(\Lambda)$.

Assume that $Z:=\overline{Z_{1} \oplus Z_{2}}$ is a strongly reduced component. Thus we have $c_{\Lambda}(Z)=E_{\Lambda}(Z)$. Applying Lemma 5.10 and Lemma 5.7(ii) this implies

$$
c_{\Lambda}\left(Z_{1}\right)+c_{\Lambda}\left(Z_{2}\right)=E_{\Lambda}\left(Z_{1}\right)+E_{\Lambda}\left(Z_{2}\right)+E_{\Lambda}\left(Z_{1}, Z_{2}\right)+E_{\Lambda}\left(Z_{2}, Z_{1}\right) .
$$

Since $c_{\Lambda}\left(Z_{i}\right) \leq E_{\Lambda}\left(Z_{i}\right)$ we get $E_{\Lambda}\left(Z_{1}, Z_{2}\right)=E_{\Lambda}\left(Z_{2}, Z_{1}\right)=0$ and $c_{\Lambda}\left(Z_{i}\right)=E_{\Lambda}\left(Z_{i}\right)$. Thus (i) implies (ii).

To show the converse, assume that $Z_{1}$ and $Z_{2}$ are strongly reduced with $E_{\Lambda}\left(Z_{1}, Z_{2}\right)=E_{\Lambda}\left(Z_{2}, Z_{1}\right)=0$. We claim that

$$
c_{\Lambda}(Z)=c_{\Lambda}\left(Z_{1}\right)+c_{\Lambda}\left(Z_{2}\right)=E_{\Lambda}\left(Z_{1}\right)+E_{\Lambda}\left(Z_{2}\right)=E_{\Lambda}(Z) .
$$

For the first equality we use Lemma 5.10 the second equality is just our assumption that $Z_{1}$ and $Z_{2}$ are strongly reduced. Finally, the third equality follows from Lemma 5.7 together with our assumption that $E_{\Lambda}\left(Z_{1}, Z_{2}\right)$ and $E_{\Lambda}\left(Z_{2}, Z_{1}\right)$ are both zero. Thus $Z$ is strongly reduced.

Note that Theorems 5.3 and 5.11 imply that each $Z \in \operatorname{decIrr}^{\text {s.r. }}(\Lambda)$ is of the form $Z=\overline{Z_{1} \oplus \cdots \oplus Z_{t}}$ with $Z_{i} \in \operatorname{dec} \operatorname{Irr}^{\text {s.r. }}(\Lambda)$ and $Z_{i}$ indecomposable for all $i$.

5.3. Generic $g$-vectors. For $Z \in \operatorname{dec} \operatorname{Irr}(\Lambda)$ there is a dense open subset $U$ of $Z$ such that $g_{\Lambda}(\mathcal{M})=g_{\Lambda}(\mathcal{N})$ for all $\mathcal{M}, \mathcal{N} \in U$. This follows again by upper semicontinuity. For $\mathcal{M} \in U$ let

$$
g_{\Lambda}(Z):=g_{\Lambda}(\mathcal{M})
$$

be the generic g-vector of $Z$.

The next lemma follows directly from upper semicontinuity and Lemma 4.1(i).

Lemma 5.12. For $Z, Z_{1}, Z_{2} \in \operatorname{dec} \operatorname{Irr}(\Lambda)$ with $Z=\overline{Z_{1} \oplus Z_{2}}$ we have

$$
g_{\Lambda}(Z)=g_{\Lambda}\left(Z_{1}\right)+g_{\Lambda}\left(Z_{2}\right) .
$$

Lemma 5.13. For $Z \in \operatorname{dec}_{\operatorname{Irr}}^{\text {s.r. }}(\Lambda)$ we have

$$
\mathbf{d} \cdot g_{\Lambda}(Z)=\operatorname{dim}(Z)-\operatorname{dim}\left(G_{\mathbf{d}}\right) .
$$


Proof. It follows from the definitions that

$$
E_{\Lambda}(Z)=\operatorname{end}_{\Lambda}(Z)+\mathbf{d} \cdot g_{\Lambda}(Z),
$$

and we have

$$
c_{\Lambda}(Z)=\operatorname{dim}(Z)-\operatorname{dim}\left(G_{\mathbf{d}}\right)+\operatorname{end}_{\Lambda}(Z) .
$$

Now the claim follows, since $c_{\Lambda}(Z)=E_{\Lambda}(Z)$.

Corollary 5.14. Let $Z \in \operatorname{dec}_{\operatorname{Irr}}^{\text {s.r. }} \mathbf{\mathbf { v }}(\Lambda)$ with $\mathbf{d} \neq 0$. If $E_{\Lambda}(Z)=0$, then

$$
\mathbf{d} \cdot g_{\Lambda}(Z)=-\operatorname{end}_{\Lambda}(Z)<0 \text {. }
$$

5.4. Parametrization of strongly reduced components. Let $\Lambda=\mathbb{C}\langle\langle Q\rangle\rangle / I$ be a finite-dimensional basic algebra. Plamondon P2 constructs a map

$$
P_{\Lambda}: \operatorname{dec} \operatorname{Irr}(\Lambda) \rightarrow \mathbb{Z}^{n},
$$

which can be defined as follows: Let $Z \in \operatorname{dec} \operatorname{Irr}(\Lambda)$. Then there exist injective $\Lambda$-modules $I_{0}^{\Lambda}(Z)$ and $I_{1}^{\Lambda}(Z)$, which are uniquely determined up to isomorphism, and a dense open subset $U \subseteq \pi Z$ such that for each representation $M \in U$ we have $I_{0}^{\Lambda}(M)=I_{0}^{\Lambda}(Z)$ and $I_{1}^{\Lambda}(M)=I_{1}^{\Lambda}(Z)$. For $Z \in \operatorname{dec}^{\prime} \operatorname{Irr}_{\mathbf{d}, \mathbf{v}}(\Lambda)$ define

$$
P_{\Lambda}(Z):=-\underline{\operatorname{dim}}\left(\operatorname{soc}\left(I_{0}^{\Lambda}(Z)\right)\right)+\underline{\operatorname{dim}}\left(\operatorname{soc}\left(I_{1}^{\Lambda}(Z)\right)\right)+\mathbf{v} .
$$

Let

$$
P_{\Lambda}^{\text {s.r. }}: \operatorname{dec}^{\text {Irr. }}{ }^{\text {s.r. }}(\Lambda) \rightarrow \mathbb{Z}^{n}
$$

be the restriction of $P_{\Lambda}$ to $\operatorname{dec} \operatorname{Irr}^{\text {s.r. }}(\Lambda)$.

For a representation $M$ let $\operatorname{add}(M)$ be the category of all finite direct sums of direct summands of $M$. Plamondon [P2] obtains the following striking result.

Theorem 5.15 (Plamondon). For any finite-dimensional basic algebra $\Lambda$ the following hold:

(i)

$$
P_{\Lambda}^{\text {s.r. }}: \operatorname{dec} \operatorname{Irr}^{\text {s.r. }}(\Lambda) \rightarrow \mathbb{Z}^{n}
$$

is bijective.

(ii) For every $Z \in \operatorname{dec} \operatorname{Irr}^{\text {s.r. }}(\Lambda)$ we have

$$
\operatorname{add}\left(I_{0}^{\Lambda}(Z)\right) \cap \operatorname{add}\left(I_{1}^{\Lambda}(Z)\right)=0 .
$$

Note that Plamondon works with irreducible components, and not with decorated irreducible components. But his results translate easily from one concept to the other.

We now generalize Theorem 5.15 (i) to arbitrary basic algebras $\Lambda$. It turns out that $\operatorname{dec} \operatorname{Irr}^{\text {s.r. }}(\Lambda)$ is in general no longer parametrized by $\mathbb{Z}^{n}$ but by a subset of $\mathbb{Z}^{n}$. Our proof is based on Plamondon's result and additionally uses truncations of basic algebras.

For a basic algebra $\Lambda$ let

$$
G_{\Lambda}: \operatorname{dec} \operatorname{Irr}(\Lambda) \rightarrow \mathbb{Z}^{n}
$$

be the map which sends $Z \in \operatorname{dec} \operatorname{Irr}(\Lambda)$ to the generic $g$-vector $g_{\Lambda}(Z)$ of $Z$.

For finite-dimensional $\Lambda$, it follows immediately from Lemma 3.3 that $G_{\Lambda}=P_{\Lambda}$. Let

$$
G_{\Lambda}^{\text {s.r. }}: \operatorname{dec}^{\text {Irr }}{ }^{\text {s.r. }}(\Lambda) \rightarrow \mathbb{Z}^{n}
$$

be the restriction of $G_{\Lambda}$ to $\operatorname{dec} \operatorname{Irr}^{\text {s.r. }}(\Lambda)$. 
For a basic algebra $\Lambda$ let

$$
\operatorname{dec} \operatorname{Irr}_{<p}(\Lambda)
$$

be the set of irreducible components $Z \in \operatorname{dec} \operatorname{Irr}(\Lambda) \operatorname{such}$ that $(\mathbf{d}, \mathbf{v}):=\underline{\operatorname{dim}}(Z)$ satisfies $|\mathbf{d}|<p$. Define

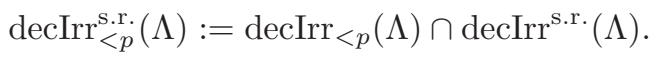

Lemma 5.16. For a basic algebra $\Lambda=\mathbb{C}\langle\langle Q\rangle\rangle / I$ the following hold:

(i) For all $p \leq q$ we have

$$
\operatorname{dec} \operatorname{Irr}_{<p}^{\text {s.r. }}\left(\Lambda_{p}\right) \subseteq \operatorname{dec}_{\operatorname{Irr}}^{\text {s.r. }}\left(\Lambda_{q}\right) \subseteq \operatorname{dec} \operatorname{Irr}^{\text {s.r. }}(\Lambda) .
$$

(ii) We have

$$
\operatorname{dec} \operatorname{Irr}^{\text {s.r. }}(\Lambda)=\bigcup_{p>0} \operatorname{dec} \operatorname{Irr}_{<p}^{\text {s.r. }}\left(\Lambda_{p}\right)
$$

(iii) We have

$$
\operatorname{dec} \operatorname{Irr}^{\text {s.r. }}(\bar{\Lambda})=\operatorname{dec} \operatorname{Irr}^{\text {s.r. }}(\Lambda) .
$$

Proof. Let $Z \in \operatorname{dec}_{\operatorname{Irr}_{\mathbf{d}, \mathbf{v}}}(\Lambda)$, and let $p>|\mathbf{d}|$. Thus we have $Z \in \operatorname{dec}_{\operatorname{Irr}_{\mathbf{d}, \mathbf{v}}}\left(\Lambda_{p}\right)$ and $Z \in \operatorname{dec}_{\operatorname{Irr}_{<p}}\left(\Lambda_{p}\right)$. Furthermore, we have $c_{\Lambda_{p}}(Z)=c_{\Lambda}(Z)$ and $E_{\Lambda_{p}}(Z)=E_{\Lambda}(Z)$. Thus $Z \in \operatorname{dec}^{\operatorname{Irr}^{\text {s.r. }}}(\Lambda)$ if and only if $Z \in \operatorname{dec}^{\text {Irr }}{ }^{\text {s.r. }}\left(\Lambda_{p}\right)$. This yields (i) and (ii). Recall that

$$
\bar{I}=\bigcap_{p \geq 0}\left(I+\mathfrak{m}^{p}\right)
$$

and $\Lambda_{p}=\mathbb{C}\langle\langle Q\rangle\rangle /\left(I+\mathfrak{m}^{p}\right)$. For $p \geq 2$ it is easy to check that

$$
\bar{I}+\mathfrak{m}^{p}=I+\mathfrak{m}^{p} .
$$

This implies $\bar{\Lambda}_{p}=\Lambda_{p}$. Now (ii) implies (iii).

Theorem 5.17. For a basic algebra $\Lambda$ the following hold:

(i) The map

$$
G_{\Lambda}^{\text {s.r. }}: \operatorname{dec} \operatorname{Irr}^{\text {s.r. }}(\Lambda) \rightarrow \mathbb{Z}^{n}
$$

is injective.

(ii) The following are equivalent:

(a) $G_{\Lambda}^{\text {s.r. }}$ is surjective.

(b) $\bar{\Lambda}$ is finite-dimensional.

Proof. Since $\Lambda_{p}$ is finite-dimensional for all $p$, we know from Plamondon's Theorem 5.15 (i) that

$$
G_{\Lambda_{p}}^{\text {s.r. }}: \operatorname{dec} \operatorname{Irr}^{\text {s.r. }}\left(\Lambda_{p}\right) \rightarrow \mathbb{Z}^{n}
$$

is bijective. Now Lemma 5.16 yields that the map

$$
G_{\Lambda}^{\text {s.r. }}: \operatorname{dec} \operatorname{Irr}^{\text {s.r. }}(\Lambda) \rightarrow \mathbb{Z}^{n}
$$

sends $Z \in \operatorname{dec} \operatorname{Irr}_{<p}^{\text {s.r. }}\left(\Lambda_{p}\right)$ to $G_{\Lambda_{p}}^{\text {s.r. }}(Z)$, and that $G_{\Lambda}^{\text {s.r. }}$ is injective. This proves (i). Theorem 5.15(i) together with Lemma 5.16(iii) says that (b) implies (a). (Recall that $\operatorname{decrep}(\Lambda)=\operatorname{decrep}(\bar{\Lambda})$. This implies that $G_{\Lambda}=G_{\bar{\Lambda}}$ and $G_{\Lambda}^{\text {s.r. }}=G_{\bar{\Lambda}}^{\text {s.r. }}$. Note also that for every $Z \in \operatorname{dec} \operatorname{Irr}(\Lambda)$ we have $G_{\Lambda}(Z)=G_{\Lambda_{p}}(Z)$ for some large enough p.)

To show the converse, assume that $\bar{\Lambda}$ is infinite-dimensional. Recall that

$$
\bar{\Lambda}=\lim _{\longleftarrow}\left(\Lambda_{p}\right)
$$


and

$$
\bar{I}_{i}=\bigcup_{p \geq 2} I_{i, p}
$$

where $\bar{I}_{i}$ is the injective envelope of the simple $\bar{\Lambda}$-module $S_{i}$, and $I_{i, p}$ is the injective envelope of $S_{i}$ considered as a $\Lambda_{p}$-module, and $\Lambda_{p}=\mathbb{C}\langle\langle Q\rangle\rangle /\left(I+\mathfrak{m}^{p}\right)$. We have $I_{i, p}=D\left(e_{i} \Lambda_{p}\right)$, where $D=\operatorname{Hom}_{K}(-, K)$ is the $K$-dual functor. This implies

$$
\operatorname{dim}\left(\Lambda_{p}\right)=\sum_{i=1}^{n} \operatorname{dim}\left(I_{i, p}\right) .
$$

It follows that there exists some $1 \leq i \leq n$ such that $\bar{I}_{i}$ is infinite-dimensional.

As a vector space, $e_{i} \Lambda_{p}$ is generated by the residue classes $a+\left(I+\mathfrak{m}^{p}\right)$ of all paths $a$ in $Q$ with $t(a)=i$. We have

$$
I_{i, 2}=D\left(e_{i} \Lambda_{2}\right)=D\left(e_{i}\left(\mathbb{C}\langle\langle Q\rangle\rangle /\left(I+\mathfrak{m}^{2}\right)\right)\right)=D\left(e_{i}\left(\mathbb{C}\langle\langle Q\rangle\rangle / \mathfrak{m}^{2}\right)\right) .
$$

This implies $\operatorname{dim}\left(I_{i, 2}\right) \geq 2$. (Otherwise, there is no arrow $a$ with $t(a)=i$, which implies $I_{i, p}=I_{i, 2}$ for all $p \geq 2$, a contradiction since $\bar{I}_{i}$ is infinite-dimensional.) Now suppose that $I_{i, p-1}=I_{i, p}$ for some $p \geq 3$. This implies $e_{i} \Lambda_{p-1}=e_{i} \Lambda_{p}$. Thus we have $e_{i}\left(I+\mathfrak{m}^{p-1}\right)=e_{i}\left(I+\mathfrak{m}^{p}\right)$. It follows that

$$
e_{i}\left(I+\mathfrak{m}^{p+1}\right)=e_{i} I+e_{i}\left(I+\mathfrak{m}^{p}\right) \mathfrak{m}=e_{i} I+e_{i}\left(I+\mathfrak{m}^{p-1}\right) \mathfrak{m}=e_{i}\left(I+\mathfrak{m}^{p}\right) .
$$

In other words, we have $I_{i, p+1}=I_{i, p}$. By induction we get $I_{i, q}=I_{i, p-1}$ for all $q \geq p$, a contradiction since $\bar{I}_{i}$ is infinite-dimensional. Thus we proved that $\operatorname{dim}\left(I_{i, p}\right) \geq p$ for all $p \geq 2$.

Now assume that $-\mathbf{e}_{i}$ is in the image of $G_{\Lambda}^{\text {s.r. }}$. (Here $\mathbf{e}_{i}$ denotes the $i$ th standard basis vector of $\mathbb{Z}^{n}$.) In other words, there is some $Z \in \operatorname{dec} \operatorname{Irr}^{\text {s.r. }}(\Lambda)$ such that $G_{\Lambda}^{\text {s.r. }}(Z)=-\mathbf{e}_{i}$. By Lemma [5.16(ii) we know that $Z \in \operatorname{dec} \operatorname{Irr}_{<p}^{\text {s.r. }}\left(\Lambda_{p}\right)$ for some $p \geq 1$. Since $g_{\Lambda}(Z)=-\mathbf{e}_{i}$, we have $I_{0}^{\Lambda_{p}}(Z)=I_{i, p}$ and $I_{1}^{\Lambda_{p}}(Z)=0$. (Here we use Theorem 5.15(ii).) This implies that $Z$ is the closure of the orbit of the decorated representation $\left(I_{i, p}, 0\right)$. But $\operatorname{dim}\left(I_{i, p}\right) \geq p$ and the dimension of all representations in $Z$ are strictly smaller than $p$, a contradiction.

\section{Component graphs and $C C$-Clusters}

6.1. The graph of strongly reduced components. Let $\Lambda$ be a basic algebra. In CBS the component graph $\Gamma(\operatorname{Irr}(\Lambda))$ of $\Lambda$ is defined as follows: The vertices of $\Gamma(\operatorname{Irr}(\Lambda))$ are the indecomposable irreducible components in $\operatorname{Irr}(\Lambda)$. There is an edge between (possibly equal) vertices $Z_{1}$ and $Z_{2}$ if $\operatorname{ext}_{\Lambda}^{1}\left(Z_{1}, Z_{2}\right)=\operatorname{ext}_{\Lambda}^{1}\left(Z_{2}, Z_{1}\right)=0$.

We want to define an analogue of $\Gamma(\operatorname{Irr}(\Lambda))$ for strongly reduced components. The graph $\Gamma\left(\right.$ decIrr $\left.^{\text {s.r. }}(\Lambda)\right)$ of strongly reduced components has as vertices the indecomposable components in $\operatorname{dec} \operatorname{Irr}^{\text {s.r. }}(\Lambda)$, and there is an edge between (possibly equal) vertices $Z_{1}$ and $Z_{2}$ if $E_{\Lambda}\left(Z_{1}, Z_{2}\right)=E_{\Lambda}\left(Z_{2}, Z_{1}\right)=0$.

6.2. Component clusters. Let $\Gamma$ be a graph, and let $\Gamma_{0}$ be the set of vertices of $\Gamma$. We allow only single edges, and we allow loops, i.e. edges from a vertex to itself. For a subset $\mathcal{U} \subseteq \Gamma_{0}$ let $\Gamma_{\mathcal{U}}$ be the full subgraph, whose set of vertices is $\mathcal{U}$. The subgraph $\Gamma_{\mathcal{U}}$ is complete if for each $i, j \in \mathcal{U}$ with $i \neq j$ there is an edge between $i$ and $j$. A complete subgraph $\Gamma_{\mathcal{U}}$ is maximal if for each complete subgraph $\Gamma_{\mathcal{U}^{\prime}}$ with $\mathcal{U} \subseteq \mathcal{U}^{\prime}$ we have $\mathcal{U}=\mathcal{U}^{\prime}$. We call a subgraph $\Gamma_{\mathcal{U}}$ loop-complete if $\Gamma_{\mathcal{U}}$ is complete and there is a loop at each vertex in $U$. 
The set of vertices of a maximal complete subgraph of $\Gamma:=\Gamma\left(\operatorname{dec} \operatorname{Irr}^{\text {s.r. }}(\Lambda)\right)$ is called a component cluster of $\Lambda$. A component cluster $\mathcal{U}$ of $\Lambda$ is E-rigid provided $E_{\Lambda}(Z)=0$ for all $Z \in \mathcal{U}$. (Recall that there is a loop at a vertex $Z$ of $\Gamma$ if and only if $E_{\Lambda}(Z, Z)=0$. If $E_{\Lambda}(Z)=0$, then $E_{\Lambda}(Z, Z)=0$, but the converse does not hold.) Clearly, each $E$-rigid component cluster is loop-complete.

Theorem 6.1. For each loop-complete subgraph $\Gamma_{\mathcal{U}}$ of $\Gamma:=\Gamma\left(\operatorname{dec} \operatorname{Irr}^{\text {s.r. }}(\Lambda)\right)$ we have $|\mathcal{U}| \leq n$.

Proof. Assume that $Z_{1}, \ldots, Z_{n+1}$ are pairwise different vertices of a loop-complete subgraph $\Gamma_{J}$ of $\Gamma\left(\operatorname{dec} \operatorname{Irr}^{\text {s.r. }}(\Lambda)\right)$. For $1 \leq i \leq n+1$ let $g_{\Lambda}\left(Z_{i}\right)$ be the generic $g$-vector of $Z_{i}$. Since $\Gamma_{J}$ is loop-complete we know by Theorem 5.11 that

$$
Z_{\mathbf{a}}:=\overline{Z_{1}^{a_{1}} \oplus \cdots \oplus Z_{n+1}^{a_{n+1}}}
$$

is again a strongly reduced component for each $\mathbf{a}=\left(a_{1}, \ldots, a_{n+1}\right) \in \mathbb{N}^{n+1}$. By the additivity of $g$-vectors we get

$$
g_{\Lambda}\left(Z_{\mathbf{a}}\right)=a_{1} g_{\Lambda}\left(Z_{1}\right)+\cdots+a_{n+1} g_{\Lambda}\left(Z_{n+1}\right) .
$$

Furthermore, we know from Theorem 5.3 that $Z_{\mathbf{a}}=Z_{\mathbf{b}}$ if and only if $\mathbf{a}=\mathbf{b}$. Now one can essentially copy the proof of [GS, Theorem 1.1] to show that there are $\mathbf{a}, \mathbf{b} \in \mathbb{N}^{n+1}$ with $g_{\Lambda}\left(Z_{\mathbf{a}}\right)=g_{\Lambda}\left(Z_{\mathbf{b}}\right)$ but $\mathbf{a} \neq \mathbf{b}$. By Theorem 5.17 different strongly reduced components have different $g$-vectors. Thus we have a contradiction.

Corollary 6.2. Let $\Lambda$ be a finite-dimensional basic algebra. Let $M$ be a representation of $\Lambda$ with $\operatorname{Hom}_{\Lambda}\left(\tau_{\Lambda}^{-}(M), M\right)=0$. Then $M$ has at most $n$ isomorphism classes of indecomposable direct summands.

The following conjecture might be a bit too optimistic, but it is true for $\Lambda=$ $\mathbb{C}\langle\langle Q\rangle\rangle$, the path algebra of an acyclic quiver $Q$; see [DW. Corollary 21] and Section 9.1

Conjecture 6.3. For any basic algebra $\Lambda$ the following hold:

(i) The component clusters of $\Lambda$ have cardinality at most $n$.

(ii) The E-rigid component clusters of $\Lambda$ are exactly the component clusters of cardinality $n$.

6.3. E-rigid representations. After most of this work was done, we learned that Iyama and Reiten [R] obtained some beautiful results on so-called $\tau$-rigid modules over finite-dimensional algebras, which fit perfectly into the framework of CalderoChapoton algebras.

Adapting their terminology to decorated representations of basic algebras, a decorated representation $\mathcal{M}$ of a basic algebra $\Lambda$ is called $E$-rigid provided $E_{\Lambda}(\mathcal{M})=0$. The following theorem is just a reformulation of Iyama and Reiten's results on $\tau$ rigid modules. Part (i) follows also directly from the more general statement in Theorem 6.1

For $\mathcal{M} \in \operatorname{decrep}(\Lambda)$ let $\Sigma(\mathcal{M})$ be the number of isomorphism classes of indecomposable direct summands of $\mathcal{M}$.

Theorem 6.4 ([R] $)$. Let $\Lambda=\mathbb{C}\langle\langle Q\rangle\rangle / I$ be a finite-dimensional basic algebra. For $\mathcal{M} \in \operatorname{decrep}(\Lambda)$ the following hold:

(i) If $\mathcal{M}$ is E-rigid, then $\Sigma(\mathcal{M}) \leq n$. 
(ii) For each E-rigid $\mathcal{M} \in \operatorname{decrep}(\Lambda)$ there exists some $\mathcal{N} \in \operatorname{decrep}(\Lambda)$ such that $\mathcal{M} \oplus \mathcal{N}$ is E-rigid and $\Sigma(\mathcal{M} \oplus \mathcal{N})=n$.

(iii) For each $E$-rigid $\mathcal{M} \in \operatorname{decrep}(\Lambda)$ with $\Sigma(\mathcal{M})=n-1$ there are exactly two non-isomorphic indecomposable decorated representations $\mathcal{N}_{1}, \mathcal{N}_{2} \in$ $\operatorname{decrep}(\Lambda)$ such that $\mathcal{M} \oplus \mathcal{N}_{i}$ is E-rigid and $\Sigma\left(\mathcal{M} \oplus \mathcal{N}_{i}\right)=n$ for $i=1,2$.

It is easy to find examples of infinite-dimensional basic algebras $\Lambda$ such that Theorem 6.4(iii) does not hold; see Section 9.3.1.

A basic algebra $\Lambda$ is representation-finite if there are only finitely many isomorphism classes of indecomposable representations in $\operatorname{rep}(\Lambda)$. One easily checks that $\Lambda$ is finite-dimensional in this case.

Corollary 6.5. Assume that $\Lambda$ is a representation-finite basic algebra. Then the following hold:

(i) Each component cluster of $\Lambda$ is E-rigid.

(ii) Each component cluster of $\Lambda$ has cardinality $n$.

(iii) There is bijection between the set of isomorphism classes of E-rigid representation of $\Lambda$ to the set $\operatorname{dec} \operatorname{Irr}^{\text {s.r. }}(\Lambda)$ of strongly reduced components. Namely, one maps an E-rigid representation $\mathcal{M}$ to the closure of the orbit $\mathcal{O}(\mathcal{M})$.

Proof. Since $\Lambda$ is representation-finite, every irreducible component $Z \in \operatorname{dec} \operatorname{Irr}(\Lambda)$ is a union of finitely many orbits, and exactly one of these orbits has do be dense in $Z$. Thus we have $c_{\Lambda}(Z)=0$. This implies (i) and (iii). Now (ii) follows directly from Theorem 6.4(ii).

6.4. Generic Caldero-Chapoton functions. For each $(\mathbf{d}, \mathbf{v}) \in \mathbb{N}^{n} \times \mathbb{N}^{n}$ let

$$
C_{\mathbf{d}, \mathbf{v}}: \operatorname{decrep}_{\mathbf{d}, \mathbf{v}}(\Lambda) \rightarrow \mathbb{Z}\left[x_{1}^{ \pm}, \ldots, x_{n}^{ \pm}\right]
$$

be the function defined by $\mathcal{M} \mapsto C_{\Lambda}(\mathcal{M})$. The map $C_{\mathbf{d}, \mathbf{v}}$ is a constructible function. In particular, the image of $C_{\mathbf{d}, \mathbf{v}}$ is finite. Thus for an irreducible component $Z \in$ $\operatorname{dec}_{\operatorname{Irr}} \mathbf{d , \mathbf { v }}(\Lambda)$ there exists a dense open subset $U \subseteq Z$ such that $C_{\mathbf{d}, \mathbf{v}}$ is constant on $U$. Define

$$
C_{\Lambda}(Z):=C_{\Lambda}(\mathcal{M})
$$

with $\mathcal{M}$ any representation in $U$. The element $C_{\Lambda}(Z)$ depends only on $Z$ and not on the choice of $U$.

Define

$$
\mathcal{B}_{\Lambda}:=\left\{C_{\Lambda}(Z) \mid Z \in \operatorname{dec}^{\text {Irr }}{ }^{\text {s.r. }}(\Lambda)\right\} .
$$

We refer to the elements of $\mathcal{B}_{\Lambda}$ as generic Caldero-Chapoton functions.

Proposition 6.6. Let $\Lambda=\mathbb{C}\langle\langle Q\rangle\rangle / I$ be a basic algebra. If $\operatorname{Ker}\left(B_{Q}\right) \cap \mathbb{Q}_{\geq 0}^{n}=0$, then $\mathcal{B}_{\Lambda}$ is linearly independent in $\mathcal{A}_{\Lambda}$.

Proof. For each $Z \in \operatorname{dec} \operatorname{Irr}^{\text {s.r. }}(\Lambda)$ there is some $\mathcal{M} \in Z$ such that $g_{\Lambda}(\mathcal{M})=g_{\Lambda}(Z)$ and $C_{\Lambda}(\mathcal{M})=C_{\Lambda}(Z)$. By Theorem 5.17 (i) the generic $g$-vectors of the strongly reduced components of decorated representations of $\Lambda$ are pairwise different. Now Proposition 4.3 yields the result.

If $\mathcal{B}_{\Lambda}$ is a basis of $\mathcal{A}_{\Lambda}$, then we call $\mathcal{B}_{\Lambda}$ the generic basis of $\mathcal{A}_{\Lambda}$. 
6.5. $C C$-clusters. For a component cluster $\mathcal{U}$ of a basic algebra $\Lambda$ let

$$
\mathcal{C}_{\mathcal{U}}:=\left\{C_{\Lambda}(Z) \mid Z \in \mathcal{U}\right\} \quad \text { and } \quad \mathcal{M}_{\mathcal{U}}:=\left\{\prod_{Z \in \mathcal{U}} C_{\Lambda}(Z)^{a_{Z}} \mid a_{Z} \in I_{Z}\right\}
$$

where

$$
I_{Z}:= \begin{cases}\mathbb{N} & \text { if } E_{\Lambda}(Z, Z)=0, \\ \{0,1\} & \text { otherwise. }\end{cases}
$$

(In each of the products above we assume that $a_{Z}=0$ for all but finitely many $Z \in \mathcal{U}$.) The set $\mathcal{C}_{\mathcal{U}}$ is called a $C C$-cluster of $\Lambda$, and the elements in $\mathcal{M}_{\mathcal{U}}$ are $C C$-cluster monomials. (The letters $C C$ just indicate that we deal with sets of Caldero-Chapoton functions.) A $C C$-cluster $\mathcal{C}_{\mathcal{U}}$ is $E$-rigid provided $E_{\Lambda}(Z)=0$ for all $Z \in \mathcal{U}$.

Note that

$$
\mathcal{C}_{\mathcal{U}} \subseteq \mathcal{M}_{\mathcal{U}} \subseteq \mathcal{A}_{\Lambda}
$$

The following result is a direct consequence of the definition of $\mathcal{B}_{\Lambda}$ and Theorem 5.11

Proposition 6.7. Let $\Lambda$ be a basic algebra. Then

$$
\mathcal{B}_{\Lambda}=\bigcup_{\mathcal{U}} \mathcal{M}_{\mathcal{U}}
$$

where the union is over all component clusters $\mathcal{U}$ of $\Lambda$.

6.6. A change of perspective. The $C C$-clusters are a generalization of the clusters of a cluster algebra defined by Fomin and Zelevinsky. In general, the FominZelevinsky cluster monomials form just a small subset of the set of $C C$-cluster monomials. Recall that the Fomin-Zelevinsky cluster monomials are obtained by the inductive procedure of cluster mutation [FZ1, FZ2, and the relation between neighbouring clusters is described by the exchange relations. One can see the exchange relations as part of the definition of a cluster algebra. On the other hand, the definition of a Caldero-Chapoton algebra does not involve any mutations of $C C$-clusters. The $C C$-clusters are given by collections of irreducible components, and they do not have to be constructed inductively. One can find a meaningful notion of neighbouring $C C$-clusters, and it remains quite a challenge to actually determine an analogue of the exchange relations.

6.7. Open problems. In this section let $\Lambda$ be any basic algebra. The following conjecture is again quite optimistic in this generality.

Conjecture 6.8. $\mathcal{B}_{\Lambda}$ is a $\mathbb{C}$-basis of $\mathcal{A}_{\Lambda}$.

Conjecture 6.8 is true for every $\Lambda=\mathbb{C}\langle\langle Q\rangle\rangle$ with $Q$ an acyclic quiver and also for numerous other examples; see GLS].

Problem 6.9. Does the set

$$
\left\{C_{\Lambda}(Z) \mid Z \in \operatorname{dec}^{\text {Irr. }}{ }^{\text {s.r. }}(\Lambda), E_{\Lambda}(Z)=0\right\}
$$

generate $\mathcal{A}_{\Lambda}$ as a $\mathbb{C}$-algebra?

We say that $\mathcal{A}_{\Lambda}$ has the Laurent phenomenon property if for any E-rigid component cluster $\left\{Z_{1}, \ldots, Z_{n}\right\}$ of $\Lambda$, we have

$$
\mathcal{A}_{\Lambda} \subseteq \mathbb{C}\left[C_{\Lambda}\left(Z_{1}\right)^{ \pm}, \ldots, C_{\Lambda}\left(Z_{n}\right)^{ \pm}\right]
$$


Problem 6.10. Under which assumptions does $\mathcal{A}_{\Lambda}$ have the Laurent phenomenon property?

\section{Caldero-Chapoton algebras and Cluster algebras}

7.1. Caldero-Chapoton algebras of Jacobian algebras. Suppose that $Q$ is a 2-acyclic quiver with a non-degenerate potential $W$, and let $\Lambda:=\mathcal{P}(Q, W)$ be the associated Jacobian algebra. Let $\mathcal{A}_{Q}$ and $\mathcal{A}_{Q}^{\text {up }}$ be the cluster algebra and upper cluster algebra associated to $Q$, respectively. Set $\mathcal{A}_{Q, W}:=\mathcal{A}_{\Lambda}, \mathcal{B}_{Q, W}:=\mathcal{B}_{\Lambda}$. Let

$$
\mathcal{M}_{Q, W}:=\left\{C_{\Lambda}(Z) \mid Z \in \operatorname{dec} \operatorname{Irr}^{\text {s.r. }}(\Lambda), E_{\Lambda}(Z)=0\right\} .
$$

The first part of the following proposition is a consequence of [DWZ2, Lemma 5.2]; compare also the calculation at the end of [GLS, Section 6.3]. The rest follows from [DWZ2, Corollary 7.2].

Proposition 7.1. We have

$$
\mathcal{A}_{Q} \subseteq \mathcal{A}_{Q, W} \subseteq \mathcal{A}_{Q}^{\text {up }}
$$

The set $\mathcal{M}_{Q}$ of cluster monomials of $\mathcal{A}_{Q}$ is contained in $\mathcal{B}_{Q, W}$. More precisely, we have

$$
\mathcal{M}_{Q} \subseteq \mathcal{M}_{Q, W} \subseteq \mathcal{B}_{Q, W}
$$

In general, the sets $\mathcal{M}_{Q}, \mathcal{M}_{Q, W}$ and $\mathcal{B}_{Q, W}$ are pairwise different.

7.2. Example. Let $Q$ be the quiver

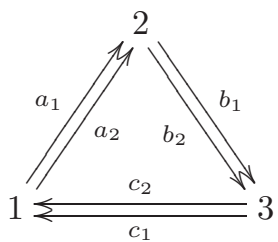

and define

$$
\begin{aligned}
& W_{1}:=c_{1} b_{1} a_{1}+c_{2} b_{2} a_{2}, \\
& W_{2}:=c_{1} b_{1} a_{1}+c_{2} b_{2} a_{2}-c_{2} b_{1} a_{2} c_{1} b_{2} a_{1} .
\end{aligned}
$$

It is not difficult to check that $\mathcal{P}\left(Q, W_{1}\right)$ is infinite-dimensional and $\mathcal{P}\left(Q, W_{2}\right)$ is finite-dimensional. By $\left[\mathrm{BFZ}\right.$, Proposition 1.26] the algebras $\mathcal{A}_{Q}$ and $\mathcal{A}_{Q}^{\text {up }}$ do not coincide. The potentials $W_{1}$ and $W_{2}$ are both non-degenerate; see [DWZ1, Example 8.6] and [La2, Example 8.2], respectively. Furthermore, by [P2, Example 4.3] the set $\mathcal{B}_{Q, W_{2}}$ of generic functions is not contained in $\mathcal{A}_{Q}$. In particular, $\mathcal{A}_{Q}$ and $\mathcal{A}_{Q, W_{2}}$ do not coincide. We conjecture that $\mathcal{A}_{Q}=\mathcal{A}_{Q, W_{1}}$ and $\mathcal{A}_{Q}^{\text {up }}=\mathcal{A}_{Q, W_{2}}$.

\subsection{Open problems.}

Problem 7.2. Are there always non-degenerate potentials $W_{1}$ and $W_{2}$ of $Q$ such that $\mathcal{A}_{Q}=\mathcal{A}_{Q, W_{1}}$ and $\mathcal{A}_{Q}^{\text {up }}=\mathcal{A}_{Q, W_{2}}$ ?

Problem 7.3. For a non-degenerate potential $W$ of $Q$ find a necessary and sufficient condition for $\mathcal{A}_{Q, W}=\mathcal{A}_{Q}^{\text {up }}$. Is this related to the two conditions

(i) $\mathcal{P}(Q, W)$ is finite-dimensional,

(ii) $W$ is rigid (see [DWZ1, Section 6] for the definition)? 
Problem 7.4. Suppose that there is only one non-degenerate potential $W$ of $Q$ up to right equivalence. Does it follows that $\mathcal{A}_{Q}=\mathcal{A}_{Q}^{\text {up }}$ ?

\section{Sign-COHEREnCE of Generic $g$-Vectors}

The following result implies Theorem 1.4. The special case where $\Lambda=\mathcal{P}(Q, W)$ is a Jacobian algebra with non-degenerate potential $W$ and $\mathcal{U}$ is an $E$-rigid component cluster is proved in $\mathrm{P} 2$, Theorem 3.7(1)].

Theorem 8.1. Let $\Lambda$ be a basic algebra, and let $\mathcal{U}$ be a component cluster of $\Lambda$. Then the set $\left\{g_{\Lambda}(Z) \mid Z \in \mathcal{U}\right\}$ is sign-coherent.

Proof. Assume that $\left\{g_{\Lambda}(Z) \mid Z \in \mathcal{U}\right\}$ is not sign-coherent. Thus there are $Z_{1}, Z_{2} \in$ $\mathcal{U}$ such that the set $\left\{g_{\Lambda}\left(Z_{1}\right), g_{\Lambda}\left(Z_{2}\right)\right\}$ is not sign-coherent. Since $\mathcal{U}$ is a component cluster, we know from Theorem 5.11 that $Z:=\overline{Z_{1} \oplus Z_{2}}$ is a strongly reduced component. By Lemma 5.12 we have $g_{\Lambda}(Z)=g_{\Lambda}\left(Z_{1}\right)+g_{\Lambda}\left(Z_{2}\right)$. By Lemma 5.16)(ii) there is some $p$ such that $Z, Z_{1}, Z_{2} \in \operatorname{dec} \operatorname{Irr}_{<p}^{\text {s.r. }}\left(\Lambda_{p}\right)$. We also know that $g_{\Lambda_{p}}(Z)=$ $g_{\Lambda}(Z)$ and $g_{\Lambda_{p}}\left(Z_{i}\right)=g_{\Lambda}\left(Z_{i}\right)$ for $i=1,2$ and that

$$
I_{0}^{\Lambda_{p}}(Z)=I_{0}^{\Lambda_{p}}\left(Z_{1}\right)+I_{0}^{\Lambda_{p}}\left(Z_{2}\right) \quad \text { and } \quad I_{1}^{\Lambda_{p}}(Z)=I_{1}^{\Lambda_{p}}\left(Z_{1}\right)+I_{1}^{\Lambda_{p}}\left(Z_{2}\right) .
$$

For $i=1,2$ let $\left(\mathbf{d}_{i}, \mathbf{v}_{i}\right):=\underline{\operatorname{dim}}\left(Z_{i}\right)$.

We first assume that $\mathbf{v}_{1}=\mathbf{v}_{2}=0$. Since $\left\{g_{\Lambda}\left(Z_{1}\right), g_{\Lambda}\left(Z_{2}\right)\right\}$ is not sign-coherent, we get from Lemma 3.4 that

$$
\operatorname{add}\left(I_{0}^{\Lambda_{p}}\left(Z_{1}\right)\right) \cap \operatorname{add}\left(I_{1}^{\Lambda_{p}}\left(Z_{2}\right)\right) \neq 0 \quad \text { or } \quad \operatorname{add}\left(I_{1}^{\Lambda_{p}}\left(Z_{1}\right)\right) \cap \operatorname{add}\left(I_{0}^{\Lambda_{p}}\left(Z_{2}\right)\right) \neq 0,
$$

a contradiction to Theorem 5.15 (ii).

Next, assume that $\mathbf{v}_{1}$ and $\mathbf{v}_{2}$ are both non-zero. The components $Z_{1}$ and $Z_{2}$ are indecomposable. It follows that $Z_{1}$ and $Z_{2}$ are just the orbits of some negative simple representations. But then $\left\{g_{\Lambda}\left(Z_{1}\right), g_{\Lambda}\left(Z_{2}\right)\right\}$ has to be sign-coherent, a contradiction.

Finally, let $\mathbf{v}_{1}=0$ and $\mathbf{v}_{2} \neq 0$. Thus we get $Z_{2}=\mathcal{O}\left(\mathcal{S}_{i}^{-}\right)$for some $1 \leq i \leq n$. This implies $g_{\Lambda}\left(Z_{2}\right)=\mathbf{e}_{i}$. Since $\left\{g_{\Lambda}\left(Z_{1}\right), g_{\Lambda}\left(Z_{2}\right)\right\}$ is not sign-coherent, the $i$ th entry of $g_{\Lambda}\left(Z_{1}\right)$ has to be negative. It follows that the socle of each representation in $Z_{1}$ has $S_{i}$ as a composition factor. In particular, the $i$ th entry $d_{i}$ of $\mathbf{d}_{1}$ is nonzero. But we also have $E_{\Lambda}\left(Z_{1}, Z_{2}\right)=0$. Now Lemma 5.4 implies that $d_{i}=0$, a contradiction.

\section{EXAmples}

\subsection{Strongly reduced components for hereditary algebras.}

9.1.1. Assume that $\Lambda=\mathbb{C}\langle\langle Q\rangle\rangle$ with $Q$ an acyclic quiver. Thus $\Lambda$ is equal to the ordinary path algebra $\mathbb{C} Q$. Clearly, for each $(\mathbf{d}, \mathbf{v}) \in \mathbb{N}^{n} \times \mathbb{N}^{n}$ the variety $\operatorname{decrep}_{\mathbf{d}, \mathbf{v}}(\Lambda)$ is an affine space. In particular, it has just one irreducible component, namely $Z_{\mathbf{d}, \mathbf{v}}:=\operatorname{decrep}_{\mathbf{d}, \mathbf{v}}(\Lambda)$.

Lemma 9.1. The following hold:

(i) For irreducible components $Z_{\mathbf{d}_{1}, 0}, Z_{\mathbf{d}_{2}, 0} \in \operatorname{dec} \operatorname{Irr}(\Lambda)$ we have

$$
\operatorname{ext}_{\Lambda}^{1}\left(Z_{\mathbf{d}_{1}, 0}, Z_{\mathbf{d}_{2}, 0}\right)=E_{\Lambda}\left(Z_{\mathbf{d}_{1}, 0}, Z_{\mathbf{d}_{2}, 0}\right) .
$$

(ii) $Z_{\mathbf{d}, \mathbf{v}}$ is strongly reduced if and only if $d_{i} v_{i}=0$ for all $1 \leq i \leq n$. 
Proof. Since $\Lambda$ is a finite-dimensional hereditary algebra, we have

$$
\operatorname{dim} \operatorname{Ext}_{\Lambda}^{1}(M, N)=\operatorname{dim} \operatorname{Hom}_{\Lambda}\left(\tau_{\Lambda}^{-}(N), M\right)
$$

for all $M, N \in \operatorname{rep}(\Lambda)$. Now Proposition 3.5 implies (i). In particular, for $Z=Z_{\mathbf{d}, 0}$ we have $e_{\Lambda}(Z)=E_{\Lambda}(Z)$. Since $Z=\operatorname{decrep}_{\mathbf{d}, 0}(\Lambda)$ is an affine space, Voigt's Lemma implies that $c_{\Lambda}(Z)=e_{\Lambda}(Z)$. Thus $Z$ is strongly reduced. The components $Z_{0, \mathbf{e}_{i}}$ are obviously also strongly reduced. Now Corollary 5.5 yields (ii).

The following result is a direct consequence of Lemma 9.1 and Schofield's Scho groundbreaking work on general representations of acyclic quivers. For all unexplained terminology we refer to [Scho.

Proposition 9.2. Let $\Lambda=\mathbb{C}\langle\langle Q\rangle\rangle$ with $Q$ an acyclic quiver. Then the indecomposable strongly reduced components are the components $Z_{\mathbf{d}, 0}$, where $\mathbf{d}$ is a Schur root, and the components $Z_{0, \mathbf{e}_{1}}, \ldots, Z_{0, \mathbf{e}_{n}}$, where $\mathbf{e}_{i}$ is the ith standard basis vector of $\mathbb{Z}^{n}$.

For a finite-dimensional path algebra $\Lambda=\mathbb{C} Q$ one can use Schofield's algorithm Scho (see also DW] for a more efficient version of the algorithm) to determine the canonical decomposition of a dimension vector, and one can also use it to decide if $\operatorname{ext}_{\Lambda}^{1}\left(Z_{1}, Z_{2}\right)$ is zero or not. So at least in principle, the graph $\Gamma\left(\operatorname{dec}^{\mathrm{Irr}}{ }^{\text {s.r. }}(\Lambda)\right)$ can be computed. However, even in this case there are numerous interesting open questions on the structure of the graph $\Gamma\left(\operatorname{dec} \operatorname{Irr}^{\text {s.r. }}(\Lambda)\right)$; see [Sche].

\subsection{Strongly reduced components for 1-vertex algebras.}

Proposition 9.3. Let $\Lambda=\mathbb{C}\langle\langle Q\rangle\rangle / I$ be a basic algebra with $n=1$. Then the following hold:

(i) If $\bar{\Lambda}$ is finite-dimensional, then the indecomposable strongly reduced components in $\operatorname{dec} \operatorname{Irr}(\Lambda)$ are $\mathcal{O}\left(\mathcal{S}_{1}^{-}\right)$and the closure of $\mathcal{O}\left(\left(\bar{I}_{1}, 0\right)\right)$, where $\bar{I}_{1}$ is the injective envelope of the simple $\bar{\Lambda}$-module $S_{1}$.

(ii) If $\bar{\Lambda}$ is infinite-dimensional, then the only indecomposable strongly reduced component in $\operatorname{dec} \operatorname{Irr}(\Lambda)$ is $\mathcal{O}\left(\mathcal{S}_{1}^{-}\right)$.

Proof. Assume that $\bar{\Lambda}$ is finite-dimensional. Then Theorem 5.17 implies that $\operatorname{Im}\left(G_{\Lambda}^{\text {s.r. }}\right)=\mathbb{Z}$. For $m \geq 0$, we know that the orbit closures of $\left(\mathcal{S}_{1}^{-}\right)^{m}$ and $\left(\bar{I}_{1}, 0\right)^{m}$ are $E$-rigid strongly reduced components with generic $g$-vectors $m \mathbf{e}_{1}$ and $-m \mathbf{e}_{1}$, respectively. This implies (i). Part (ii) follows from the proof of Theorem5.17(ii).

\subsection{Strongly reduced components for some representation-finite alge-}

bras. By Corollary 6.5 each vertex of the component graph of a representationfinite basic algebra has a loop. In the following examples, for each $E$-rigid indecomposable strongly reduced component, we just display the indecomposable decorated representation whose orbit closure is the component. We describe representations by displaying their socle series and their composition factors. For $1 \leq i \leq n$ we write $i$ and $-i$ instead of $\mathcal{S}_{i}$ and $\mathcal{S}_{i}^{-}$, respectively. For a decorated representation of the form $\mathcal{M}=(M, 0)$ we just display $M$. 
9.3.1. Let $Q$ be the quiver

$$
1 \leftarrow \text { b } 2 \bigcirc a
$$

and let $\Lambda:=\mathbb{C}\langle\langle Q\rangle\rangle / I$, where $I$ is generated by $b a$. Then $\Gamma\left(\operatorname{dec} \operatorname{Irr}^{\text {s.r. }}(\Lambda)\right)$ looks as follows:

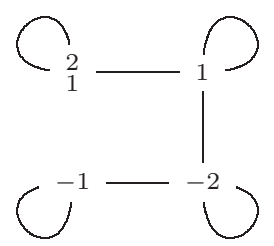

For $p=2$, the component graph $\Gamma\left(\operatorname{dec} \operatorname{Irr}^{\text {s.r. }}\left(\Lambda_{p}\right)\right)$ looks as follows:

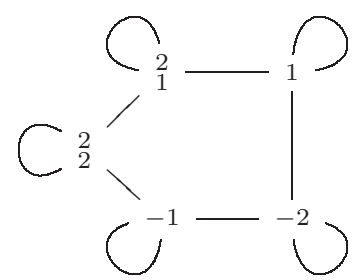

To repair the somewhat non-symmetric graph $\Gamma\left(\operatorname{dec} \operatorname{Irr}^{\text {s.r. }}(\Lambda)\right)$ one could insert a vertex for the infinite-dimensional indecomposable injective $\Lambda$-module $I_{2}$. Such aspects will be dealt with elsewhere.

9.3.2. Let $Q$ be the quiver

$$
1 \leftarrow^{b} 2 \leftarrow^{a}-3
$$

and let $\Lambda:=\mathbb{C}\langle\langle Q\rangle\rangle / I$, where $I$ is generated by ba. Then $\Gamma\left(\operatorname{dec} \operatorname{Irr}^{\text {s.r. }}(\Lambda)\right)$ looks as follows:

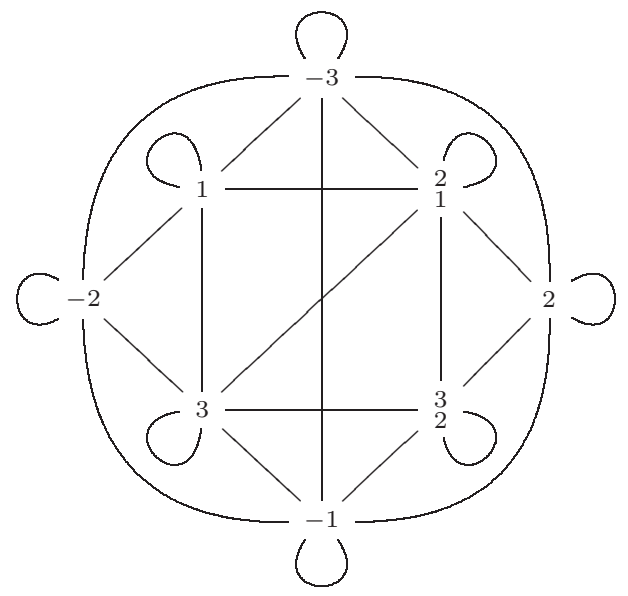

Note that for $M={ }_{2}^{3}$ and $N=1$ we have $\operatorname{Ext}_{\Lambda}^{1}(M, N)=0$, but $E_{\Lambda}((M, 0),(N, 0))$ $=\operatorname{Hom}_{\Lambda}\left(\tau^{-}(N), M\right) \neq 0$. 


\subsection{Examples of Caldero-Chapoton algebras.}

9.4.1. Let $Q$ be the quiver

$$
1 \circlearrowleft
$$

and let $\Lambda:=\mathbb{C}\langle\langle Q\rangle\rangle$. The skew-symmetric adjacency matrix $B_{Q}$ of $Q$ is just (0). $\mathrm{Up}$ to isomorphism, for each $d \geq 1$ there is a unique indecomposable representation $M_{d}$ of $\Lambda$ with $\operatorname{dim}\left(M_{d}\right)=d$, given by a nilpotent Jordan block of size $d$. One easily checks that

$$
C_{\Lambda}\left(M_{d}\right)=d+1
$$

This implies $\mathcal{A}_{\Lambda}=\mathbb{C}$.

For $p \geq 2$ the indecomposable representations of the $p$-truncation $\Lambda_{p}$ are $M_{1}, \ldots$, $M_{p}$, and we get

$$
C_{\Lambda_{p}}\left(M_{d}\right)= \begin{cases}(d+1) x_{1}^{-1} & \text { if } d=p, \\ (d+1) & \text { otherwise }\end{cases}
$$

This implies $\mathcal{A}_{\Lambda_{p}}=\mathbb{C}\left[x_{1}^{-1}\right]$.

9.4.2. In this section, let $Q$ be the quiver

$$
1 \stackrel{a}{\longrightarrow} 2 \stackrel{b}{\longrightarrow} 3 \fallingdotseq c
$$

and let $\Lambda:=\mathbb{C}\langle\langle Q\rangle\rangle / I$, where $I$ is the ideal generated by $c^{2}$. (This example is closely related to Zhou and Zhu's [ZZ] study of cluster tubes. We thank the anonymous referee for pointing this out. The considerations in this section also hold for the corresponding more general case of a linear quiver with $n$ vertices and a loop $c$ with $c^{2}=0$. This and also a comparison with the results in $[\mathrm{ZZ}]$ will be carried out in a separate publication.)

The basic algebra $\Lambda$ is a representation-finite string algebra, and its AuslanderReiten quiver looks as follows: (Recall that there is an arrow from $M$ to $N$ if and only if there is an irreducible homomorphism from $M$ to $N$, and for all nonprojective $M$ we draw a dashed arrow from $M$ to its Auslander-Reiten translate $\tau_{\Lambda}(M)$.)

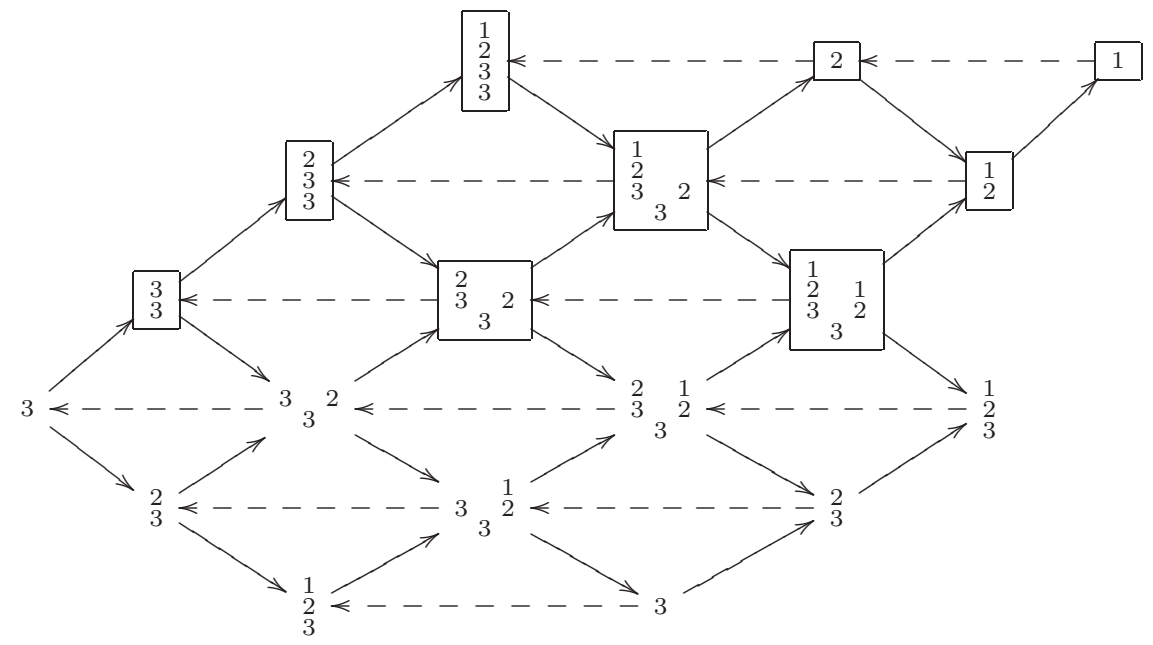


In this quiver the two south-west and the two south-east edges are identified. The framed representations are the indecomposable $E$-rigid representations of $\Lambda$. We have

$$
I_{1}=1, \quad I_{2}=\frac{1}{2}, \quad I_{3}={ }_{3}^{2} \underset{3}{2} .
$$

We now describe explicitly the Caldero-Chapoton functions associated to the 12 indecomposable $E$-rigid decorated representations of $\Lambda$. By definition $C_{\Lambda}\left(\mathcal{S}_{i}^{-}\right)=x_{i}$, for $i=1,2,3$. The remaining 9 functions are

$$
\begin{aligned}
& C_{\Lambda}(1)=\frac{1+x_{2}}{x_{1}}, \quad C_{\Lambda}\left(\begin{array}{l}
1 \\
2
\end{array}\right)=\frac{x_{1}+x_{3}+x_{2} x_{3}}{x_{1} x_{2}}, \\
& C_{\Lambda}\left(\begin{array}{c}
1 \\
2 \\
3 \\
3
\end{array}\right)=\frac{x_{1} x_{2}^{2}+x_{1} x_{2}+x_{1}+x_{3}+x_{2} x_{3}}{x_{1} x_{2} x_{3}}, \quad C_{\Lambda}(2)=\frac{x_{1}+x_{3}}{x_{2}}, \\
& C_{\Lambda}\left(\begin{array}{l}
3 \\
3
\end{array}\right)=\frac{x_{2}^{2}+x_{2}+1}{x_{3}}, \quad C_{\Lambda}\left(\begin{array}{c}
2 \\
3 \\
3
\end{array}\right)=\frac{x_{1} x_{2}^{2}+x_{1} x_{2}+x_{1}+x_{3}}{x_{2} x_{3}},
\end{aligned}
$$

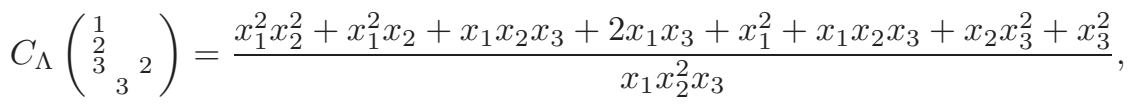

$$
\begin{aligned}
& C_{\Lambda}\left(\begin{array}{ll}
1 & 1 \\
2 & 1 \\
3 & 2 \\
& 3
\end{array}\right) \\
& =\frac{x_{1}^{2} x_{2}^{2}+x_{1}^{2} x_{2}+x_{1}^{2}+x_{1} x_{2} x_{3}+2 x_{1} x_{3}+x_{3}^{2}+x_{1} x_{2}^{2} x_{3}+2 x_{1} x_{2} x_{3}+2 x_{2} x_{3}^{2}+x_{2}^{2} x_{3}^{2}}{x_{1}^{2} x_{2}^{2} x_{3}}, \\
& C_{\Lambda}\left({ }_{3}^{2}{ }_{3}^{2}\right)=\frac{x_{1}^{2} x_{2}^{2}+x_{1}^{2} x_{2}+x_{1}^{2}+x_{1} x_{2} x_{3}+2 x_{1} x_{3}+x_{3}^{2}}{x_{2}^{2} x_{3}} .
\end{aligned}
$$

The Caldero-Chapoton functions associated to the 6 indecomposable non- $E$-rigid representations of $\Lambda$ are

$$
\begin{aligned}
C_{\Lambda}(3) & =x_{2}+1, \\
C_{\Lambda}\left(\begin{array}{l}
2 \\
3
\end{array}\right) & =\frac{x_{1} x_{2}+x_{1}+x_{3}}{x_{2}}, \\
C_{\Lambda}\left({ }_{3}^{3}{ }_{3}^{2}\right) & =\frac{x_{1} x_{2}^{2}+x_{1} x_{2}+x_{1}+x_{3}+x_{2} x_{3}}{x_{2} x_{3}}, \\
C_{\Lambda}\left(\begin{array}{l}
1 \\
2 \\
3
\end{array}\right) & =\frac{x_{1} x_{2}+x_{1}+x_{3}+x_{2} x_{3}}{x_{1} x_{2}}, \\
C_{\Lambda}\left(\begin{array}{r}
1 \\
3
\end{array}{ }^{2}\right) & =\frac{x_{1} x_{2}^{2}+x_{1} x_{2}+x_{1}+x_{2} x_{3}+x_{2}^{2} x_{3}+x_{2} x_{3}+x_{3}}{x_{1} x_{2} x_{3}}, \\
C_{\Lambda}\left(\begin{array}{rr}
2 & 1 \\
3 & 2
\end{array}\right) & =\frac{x_{1}^{2} x_{2}^{2}+x_{1}^{2} x_{2}+x_{1}^{2}+x_{1} x_{2} x_{3}+2 x_{1} x_{3}+x_{3}^{2}+x_{1} x_{2}^{2} x_{3}+x_{1} x_{2} x_{3}+x_{2} x_{3}^{2}}{x_{1} x_{2}^{2} x_{3}} .
\end{aligned}
$$

The following statement says that in our example, there is a positive answer to Problem 6.9.

Proposition 9.4. The set

$$
\left\{C_{\Lambda}(Z) \mid Z \in \operatorname{dec}^{\text {Irr.r. }}(\Lambda), E_{\Lambda}(Z)=0\right\}
$$

generates the Caldero-Chapoton algebra $\mathcal{A}_{\Lambda}$ as a $\mathbb{C}$-algebra. 
Proof. It is enough to express the Caldero-Chapoton functions of the 6 indecomposable non- $E$-rigid representations in terms of Caldero-Chapoton functions of $E$-rigid decorated representations. An easy calculation yields

$$
\begin{aligned}
& C_{\Lambda}(3)=x_{2}+1, \\
& C_{\Lambda}\left(\begin{array}{l}
2 \\
3
\end{array}\right)=x_{1}+C_{\Lambda}(2), \\
& C_{\Lambda}\left(\begin{array}{ll}
3{ }_{3} & 2
\end{array}\right)=C_{\Lambda}\left(\begin{array}{l}
2 \\
3 \\
3
\end{array}\right)+1 \\
& C_{\Lambda}\left(\begin{array}{l}
1 \\
2 \\
3
\end{array}\right)=C_{\Lambda}\left(\begin{array}{l}
1 \\
2
\end{array}\right)+1, \\
& C_{\Lambda}\left(\begin{array}{ll}
3 & \frac{1}{2} \\
& 3
\end{array}\right)=C_{\Lambda}\left(\begin{array}{l}
1 \\
2 \\
3 \\
3
\end{array}\right)+C_{\Lambda}(1), \\
& C_{\Lambda}\left(\begin{array}{ll}
2 & 1 \\
3 & 2 \\
& 3
\end{array}\right)=C_{\Lambda}\left(\begin{array}{ll}
1 & \\
2 & \\
3 & 2
\end{array}\right)+1 .
\end{aligned}
$$

All summands of the right hand side of the above equations are Caldero-Chapoton functions of $E$-rigid decorated representations, and the 6 Caldero-Chapoton functions of the indecomposable non- $E$-rigid representations are on the left side. (Note that $x_{i}=C_{\Lambda}\left(\mathcal{S}_{i}^{-}\right)$and $1=C_{\Lambda}(0)$, and $\mathcal{S}_{i}^{-}$and 0 are both $E$-rigid.) This finishes the proof.

Since $\Lambda$ is representation-finite, each strongly reduced component contains an $E$-rigid decorated representation. Each vertex of $\Gamma\left(\operatorname{dec} \operatorname{Irr}^{\text {s.r. }}(\Lambda)\right)$ has a loop. Let $\Gamma\left(\operatorname{dec} \operatorname{Irr}^{\text {s.r. }}(\Lambda)\right)^{\circ}$ be the graph obtained by deleting these loops. We display $\Gamma\left(\operatorname{dec} \operatorname{Irr}^{\text {s.r. }}(\Lambda)\right)^{\circ}$ in the following picture. Note that each component cluster is $E$-rigid and contains exactly three irreducible components.

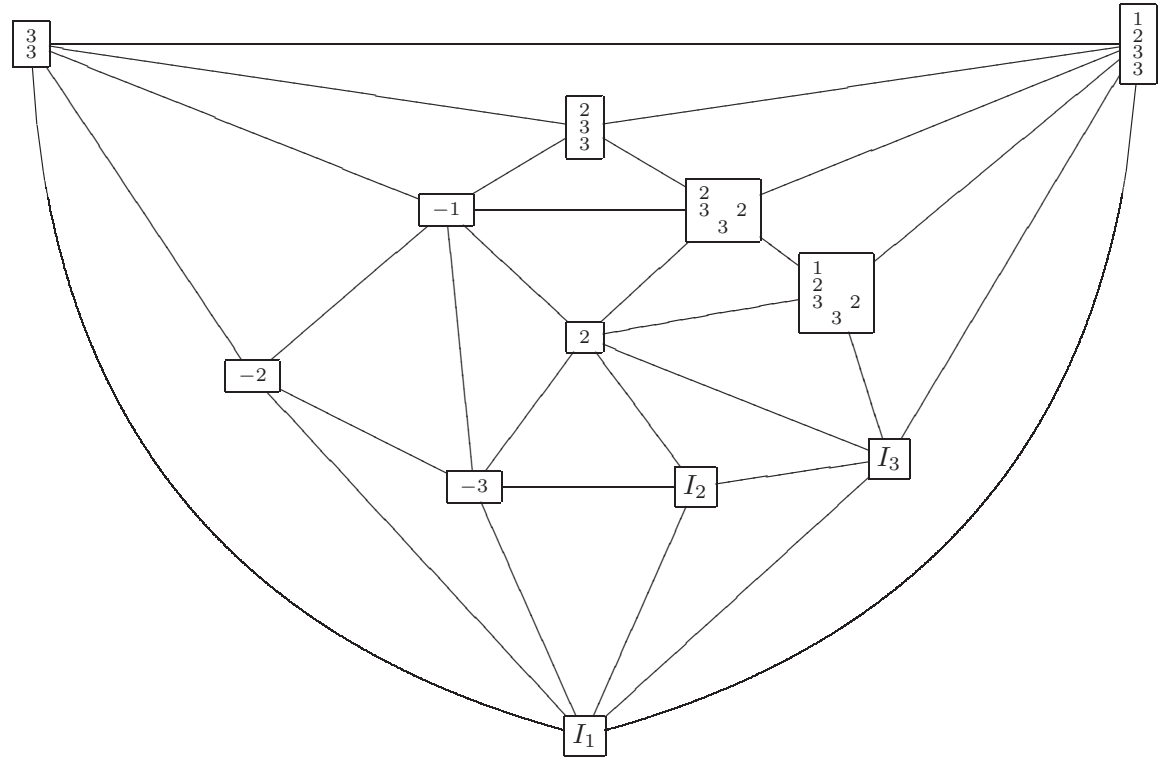

9.4.3. Let $Q$ be the 2 -Kronecker quiver 
and let $\Lambda=\mathbb{C}\langle\langle Q\rangle\rangle$. The following picture describes the quiver $\Gamma\left(\operatorname{dec}^{\mathrm{Ir} r}{ }^{\mathrm{s.r} .}(\Lambda)\right)$. (For indecomposable strongly reduced components of the form $Z_{\mathbf{d}, 0}$ or $Z_{0, \mathbf{e}_{i}}$ we just display the vectors $\mathbf{d}$ or $-\mathbf{e}_{i}$, respectively.)

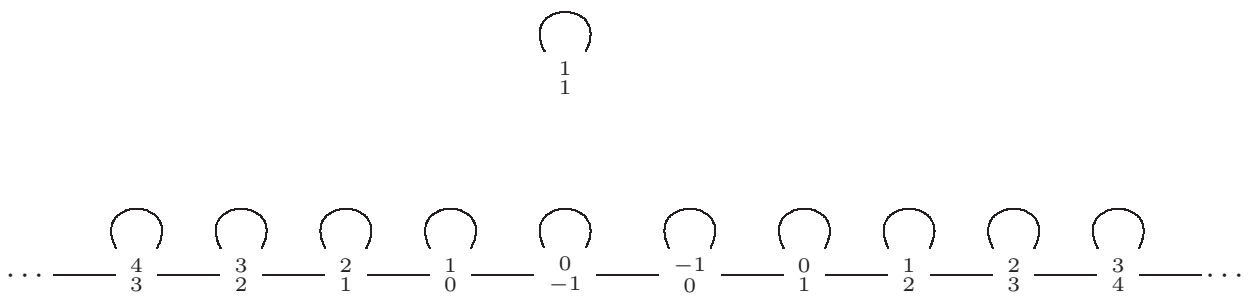

Thus there is exactly one component cluster $\{Z\}$ of cardinality one, and there are infinitely many component clusters of cardinality two. One can easily check that $E_{\Lambda}(Z, Z)=0$, hence the loop at $Z$, but $E_{\Lambda}(Z) \neq 0$. Thus $\{Z\}$ is not $E$-rigid. The other component clusters are $E$-rigid. The $C C$-cluster monomials are

$$
C_{\Lambda}\left(\begin{array}{c}
0 \\
-1
\end{array}\right)^{a} C_{\Lambda}\left(\begin{array}{c}
-1 \\
0
\end{array}\right)^{b}, \quad C_{\Lambda}\left(\begin{array}{c}
i+1 \\
i
\end{array}\right)^{a} C_{\Lambda}\left(\begin{array}{c}
i \\
i-1
\end{array}\right)^{b}, \quad C_{\Lambda}\left(\begin{array}{c}
i-1 \\
i
\end{array}\right)^{a} C_{\Lambda}\left(\begin{array}{c}
i \\
i+1
\end{array}\right)^{b} \quad \text { and } \quad C_{\Lambda}\left(\begin{array}{l}
1 \\
1
\end{array}\right)^{a}
$$

where $a, b, i \geq 0$.

The set $\mathcal{B}_{\Lambda}$ of generic Caldero-Chapoton functions is just the set of $C C$-cluster monomials. Recall from [BFZ that for any acyclic quiver $Q$ we have $\mathcal{A}_{Q}=\mathcal{A}_{Q}^{\text {up }}$. In this case, $\mathcal{B}_{\Lambda}$ is a $\mathbb{C}$-basis of $\mathcal{A}_{Q}$; see GLS.

For acyclic quivers $Q$ of wild representation type and $\Lambda=\mathbb{C}\langle\langle Q\rangle\rangle$, the component graph $\Gamma\left(\operatorname{dec} \operatorname{Irr}^{\text {s.r. }}(\Lambda)\right)$ will have vertices without loops. For example, let $Q$ be the 3 -Kronecker quiver

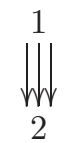

and let $\Lambda=\mathbb{C}\langle\langle Q\rangle\rangle$. Let

$$
\phi=\left(\begin{array}{ll}
-1 & 3 \\
-3 & 8
\end{array}\right)
$$

be the Coxeter matrix of $\Lambda$. For $k \geq 0$ define

$$
\begin{aligned}
\mathbf{p}_{2 k} & :=\phi^{k}\left(\begin{array}{l}
0 \\
1
\end{array}\right), \\
\mathbf{p}_{2 k+1} & :=\phi^{k}\left(\begin{array}{l}
1 \\
3
\end{array}\right), \\
\mathbf{q}_{2 k} & :=\phi^{-k}\left(\begin{array}{l}
1 \\
0
\end{array}\right), \\
\mathbf{q}_{2 k+1} & :=\phi^{-k}\left(\begin{array}{l}
3 \\
1
\end{array}\right) .
\end{aligned}
$$

Set $\mathbf{p}_{-1}:=-\mathbf{e}_{1}$ and $\mathbf{q}_{-1}:=-\mathbf{e}_{2}$. One connected component of the component graph $\Gamma\left(\operatorname{dec} \operatorname{Irr}^{\text {s.r. }}(\Lambda)\right)$ looks as follows:

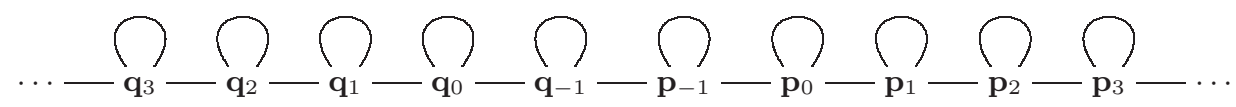

These are precisely the $E$-rigid vertices of $\Gamma\left(\operatorname{dec} \operatorname{Irr}^{\text {s.r. }}(\Lambda)\right)$. 
The set of Schur roots of $Q$ consists of real and imaginary Schur roots. The above picture shows the real Schur roots (and the vectors $-\mathbf{e}_{1}$ and $-\mathbf{e}_{2}$ ). The set $R_{\mathrm{im}}^{+}$of imaginary Schur roots consists of all dimension vectors $\mathbf{d}=\left(d_{1}, d_{2}\right) \in \mathbb{N}^{2}$ with $d_{2} \neq 0$ such that

$$
(3-\sqrt{5}) / 2 \leq d_{1} / d_{2} \leq(3+\sqrt{5}) / 2
$$

see [DW, Section 3] and [K, Section 6]. There is no edge between $Z_{\mathbf{d}, 0}$ and any other vertex of $\Gamma\left(\operatorname{dec} \operatorname{Irr}^{\text {s.r. }}(\Lambda)\right)$. In particular, there is no loop at $Z_{\mathbf{d}, 0}$.

The $C C$-cluster monomials are

$$
\begin{gathered}
C_{\Lambda}\left(\mathbf{q}_{-1}\right)^{a} C_{\Lambda}\left(\mathbf{p}_{-1}\right)^{b}, \quad C_{\Lambda}\left(\mathbf{p}_{i-1}\right)^{a} C_{\Lambda}\left(\mathbf{p}_{i}\right)^{b}, \\
C_{\Lambda}\left(\mathbf{q}_{i}\right)^{a} C_{\Lambda}\left(\mathbf{q}_{i-1}\right)^{b} \quad \text { and } \quad C_{\Lambda}(\mathbf{d})
\end{gathered}
$$

where $a, b, i \geq 0$ and $\mathbf{d} \in R_{\mathrm{im}}^{+}$. Again it follows from [GLS that these $C C$-cluster monomials form a $\mathbb{C}$-basis of $\mathcal{A}_{Q}$. It remains a challenge to compute the exchange relations between all neighbouring $C C$-clusters. For the $E$-rigid $C C$-clusters, the exchange relations are known from the Fomin-Zelevinsky exchange relations arising from mutations of clusters. But for $\mathbf{d}, \mathbf{d}_{1}, \mathbf{d}_{2} \in R_{\mathrm{im}}^{+}$and $i \geq-1$ it remains an open problem to express the products

$$
C_{\Lambda}(\mathbf{d}) C_{\Lambda}\left(\mathbf{p}_{i}\right), \quad C_{\Lambda}(\mathbf{d}) C_{\Lambda}\left(\mathbf{q}_{i}\right) \quad \text { and } \quad C_{\Lambda}\left(\mathbf{d}_{1}\right) C_{\Lambda}\left(\mathbf{d}_{2}\right)
$$

as linear combinations of elements from the basis $\mathcal{B}_{\Lambda}$.

\section{ACKNOWLEDGEMENTS}

The authors thank Ben Davison, Christof Geiß, Sven Meinhardt and PierreGuy Plamondon for helpful discussions. The authors are indebted to Charlotte Ricke for carefully reading the first version of this article and for pointing out some inaccuracies.

\section{REFERENCES}

[A] Claire Amiot, Cluster categories for algebras of global dimension 2 and quivers with potential (English, with English and French summaries), Ann. Inst. Fourier (Grenoble) 59 (2009), no. 6, 2525-2590. MR2640929(2011c:16026)

[AR] Maurice Auslander and Idun Reiten, Modules determined by their composition factors, Illinois J. Math. 29 (1985), no. 2, 280-301. MR784524(86i:16032)

[ARS] Maurice Auslander, Idun Reiten, and Sverre O. Smalø, Representation theory of Artin algebras, Cambridge Studies in Advanced Mathematics, vol. 36, Cambridge University Press, Cambridge, 1997. Corrected reprint of the 1995 original. MR1476671 (98e:16011)

[ASS] Ibrahim Assem, Daniel Simson, and Andrzej Skowroński, Elements of the representation theory of associative algebras. Vol. 1, Techniques of representation theory. London Mathematical Society Student Texts, vol. 65, Cambridge University Press, Cambridge, 2006. MR2197389 (2006j:16020)

[BFZ] Arkady Berenstein, Sergey Fomin, and Andrei Zelevinsky, Cluster algebras. III. Upper bounds and double Bruhat cells, Duke Math. J. 126 (2005), no. 1, 1-52, DOI 10.1215/S0012-7094-04-12611-9. MR2110627(2005i:16065) 
[CC] Philippe Caldero and Frédéric Chapoton, Cluster algebras as Hall algebras of quiver representations, Comment. Math. Helv. 81 (2006), no. 3, 595-616, DOI 10.4171/CMH/65. MR2250855(2008b:16015)

[C] Giovanni Cerulli Irelli, Cluster algebras of type $A_{2}^{(1)}$, Algebr. Represent. Theory 15 (2012), no. 5, 977-1021, DOI 10.1007/s10468-011-9275-5. MR2969285

[CBS] William Crawley-Boevey and Jan Schröer, Irreducible components of varieties of modules, J. Reine Angew. Math. 553 (2002), 201-220, DOI 10.1515/crll.2002.100. MR:1944812(2004a:16020)

[DW] Harm Derksen and Jerzy Weyman, On the canonical decomposition of quiver representations, Compositio Math. 133 (2002), no. 3, 245-265, DOI 10.1023/A:1020007100426. MR:1930979 (2003h:16017)

[DWZ1] Harm Derksen, Jerzy Weyman, and Andrei Zelevinsky, Quivers with potentials and their representations. I. Mutations, Selecta Math. (N.S.) 14 (2008), no. 1, 59-119, DOI 10.1007/s00029-008-0057-9. MR2480710 (2010b:16021)

[DWZ2] Harm Derksen, Jerzy Weyman, and Andrei Zelevinsky, Quivers with potentials and their representations. II: Applications to cluster algebras, J. Amer. Math. Soc. 23 (2010), no. 3, 749-790, DOI 10.1090/S0894-0347-10-00662-4. MR2629987(2012c:16044)

[FZ1] Sergey Fomin and Andrei Zelevinsky, Cluster algebras. I. Foundations, J. Amer. Math. Soc. 15 (2002), no. 2, 497-529 (electronic), DOI 10.1090/S0894-0347-01-00385X. MR 1887642 (2003f:16050)

[FZ2] Sergey Fomin and Andrei Zelevinsky, Cluster algebras. II. Finite type classification, Invent. Math. 154 (2003), no. 1, 63-121, DOI 10.1007/s00222-003-0302-y. MR2004457 (2004m:17011)

[FK] Changjian Fu and Bernhard Keller, On cluster algebras with coefficients and 2-CalabiYau categories, Trans. Amer. Math. Soc. 362 (2010), no. 2, 859-895, DOI 10.1090/S00029947-09-04979-4. MR2551509 (2011b:13076)

[G] Peter Gabriel, Finite representation type is open, Proceedings of the International Conference on Representations of Algebras (Carleton Math. Lecture Notes, No. 9), Carleton Univ., Ottawa, Ont., 1974, pp. 23. MR0376769 (51 \#12944)

[GLaS] C. Geiß, D. Labardini-Fragoso, and J. Schröer, preprint in preparation.

[GLS] Christof Geiss, Bernard Leclerc, and Jan Schröer, Generic bases for cluster algebras and the Chamber ansatz, J. Amer. Math. Soc. 25 (2012), no. 1, 21-76, DOI 10.1090/S08940347-2011-00715-7. MR2833478 (2012g:13041)

[GS] Christof Geiss and Jan Schröer, Extension-orthogonal components of preprojective varieties, Trans. Amer. Math. Soc. 357 (2005), no. 5, 1953-1962 (electronic), DOI 10.1090/S0002-9947-04-03555-X. MR2115084(2006c:16023)

[IR] O. Iyama and I. Reiten, $\tau$-tilting modules, talk in Trondheim on 28.03.2012.

[K] V. G. Kac, Infinite root systems, representations of graphs and invariant theory. II, J. Algebra 78 (1982), no. 1, 141-162, DOI 10.1016/0021-8693(82)90105-3. MR677715 (85b:17003)

[La1] Daniel Labardini-Fragoso, Quivers with potentials associated to triangulated surfaces, Proc. Lond. Math. Soc. (3) 98 (2009), no. 3, 797-839, DOI 10.1112/plms/pdn051. MR:2500873(2010b:16033)

[La2] D. Labardini-Fragoso, Quivers with potentials associated to triangulated surfaces, Part II: Arc representations, Preprint (2009), 52pp., arXiv:0909.4100v2

[MRZ] Robert Marsh, Markus Reineke, and Andrei Zelevinsky, Generalized associahedra via quiver representations, Trans. Amer. Math. Soc. 355 (2003), no. 10, 4171-4186, DOI 10.1090/S0002-9947-03-03320-8. MR:1990581 (2004g:52014)

[Pa] Yann Palu, Cluster characters for 2-Calabi-Yau triangulated categories (English, with English and French summaries), Ann. Inst. Fourier (Grenoble) 58 (2008), no. 6, 22212248. MR2473635(2009k:18013)

[P1] Pierre-Guy Plamondon, Cluster algebras via cluster categories with infinitedimensional morphism spaces, Compos. Math. 147 (2011), no. 6, 1921-1934, DOI 10.1112/S0010437X11005483. MR2862067

[P2] P.-G. Plamondon, Generic bases for cluster algebras from the cluster category, Int. Math. Res. Notices IMRN 2013, no. 10, 2368-2420. MR3061943

[Sche] S. Scherotzke, Generalized clusters for acyclic quivers, preprint (2012). 
[Scho] Aidan Schofield, General representations of quivers, Proc. London Math. Soc. (3) 65 (1992), no. 1, 46-64, DOI 10.1112/plms/s3-65.1.46. MR1162487 (93d:16014)

[ZZ] Y. Zhou and B. Zhu, Cluster algebras arising from cluster tubes, J. London Math. Soc. (2) 89 (2014), no. 3, 703-723. MR3217645

Mathematisches Institut, Universität Bonn, Endenicher Allee 60, 53115 Bonn, GerMANY

E-mail address: cerulli.math@googlemail.com

Mathematisches Institut, Universität Bonn, Endenicher Allee 60, 53115 Bonn, GerMANY

E-mail address: labardini@matem.unam.mx

Mathematisches Institut, Universität Bonn, Endenicher Allee 60, 53115 Bonn, GerMANY

E-mail address: schroer@math.uni-bonn.de 